\title{
Adaptive OFDM-IM for Enhancing Physical Layer Security and Spectral Efficiency of Future Wireless Networks
}

\author{
Haji M. Furqan $\mathbb{D}^{1},{ }^{1}$ Jehad M. Hamamreh $\mathbb{D}{ }^{1}$ and Huseyin Arslan $\mathbb{D}^{1,2}$ \\ ${ }^{1}$ Department of Electrical and Electronics Engineering, Istanbul Medipol University, Istanbul 34810, Turkey \\ ${ }^{2}$ Department of Electrical Engineering, University of South Florida, Tampa, FL 33620, USA \\ Correspondence should be addressed to Haji M. Furqan; hamadni@st.medipol.edu.tr
}

Received 27 April 2018; Revised 7 July 2018; Accepted 31 July 2018; Published 15 August 2018

Academic Editor: Lu Wei

Copyright (c) 2018 Haji M. Furqan et al. This is an open access article distributed under the Creative Commons Attribution License, which permits unrestricted use, distribution, and reproduction in any medium, provided the original work is properly cited.

\begin{abstract}
In this paper, we propose algorithms for enhancing physical layer security and spectral efficiency of Orthogonal Frequency Division Multiplexing (OFDM) with Index Modulation (IM) systems. Particularly, different activation ratios and/or Constellation Modulation orders are selected adaptively for each subblock based on the channel quality of the legitimate receiver. More specifically, three approaches named as (1) OFDM with Adaptive Index Modulation and Fixed Constellation Modulation (OFDM-AIM-FCM), (2) OFDM with Adaptive Index Modulation and Adaptive Constellation Modulation (OFDM-AIM-ACM), and (3) OFDM with Variable Index Modulation and Variable Constellation Modulation (OFDM-VIM-VCM) are proposed for enhancing physical layer security and spectral efficiency. Simulation results are presented to investigate the effectiveness of the proposed algorithms.
\end{abstract}

\section{Introduction}

The inherent broadcast characteristic of wireless communication makes it vulnerable to the passive eavesdropping. Conventionally, security techniques in the upper layers, such as cryptography based techniques, have been employed for secure transmission. However, such security techniques may not be adequate for future decentralized networks due to their high complexity in implementation and computation [1]. Furthermore, the emergence of powerful computing devices makes these techniques vulnerable to sophisticated adversaries. In order to cope up with these problems, Physical Layer Security (PLS) techniques have attracted a lot of attentions [2]. PLS techniques exploit the dynamic features of wireless communications, such as channel randomness, interference, and noise, to prevent the eavesdropper from decoding data while ensuring that the legitimate user can decode it successfully [1]. In the literature, practical signal processing based PLS techniques are proposed in order to secure communication between legitimate parties $[3,4]$.

On the other hand, Index Modulation (IM) is an emerging technique for future wireless networks because of its higher energy efficiency (EE) and controllable spectral efficiency (SE) [5]. Spatial Modulation (SM) and OFDM-IM are two well-known applications. OFDM-IM especially, has been studied intensively in the literature $[5,6]$. Unlike conventional OFDM, which sends data via $\mathrm{M}$-ary signal constellation, in OFDM-IM, data is sent by both M-ary signal constellation and indices of the subcarriers. Due to high EE and adjustable SE, it is considered not only for Machine Type Communication (MTC) but also for high speed wireless communication systems [5, 7]. There are a lot of interesting works for enhancing spectral efficiency of SM and OFDMIM. In [8], precoding based technique is proposed in which spatial modulation works in both the in-phase and quadrature parts of the received signals, thus conveying additional information bits compared with conventional generalized precoding-aided spatial modulation. In [9], information is conveyed through multiple distinguishable modes and their full permutations. Authors proposed frequency Index Modulation technique and a joint code-frequency Index Modulation techniques for enhancing energy and spectral efficiency in [10,11], respectively. The proposed techniques are simple and can reduce PAPR without sacrificing data rate. In [12], authors proposed a scheme to enhance the spectral and energy efficiency by using initial conditions to generate different chaotic sequences that can convey extra bits per transmission. 
In the following, we will first explain some of the related and popular PLS techniques for OFDM and then for SM and finally for OFDM-IM. In the literature, many promising PLS techniques have been proposed for OFDM. In [13], secret key generation based techniques are proposed for OFDM system. The basic idea is to extract random sequence from the wireless channel. Motivated by the effectiveness of Artificial Noise (AN) for providing PLS, authors in [14] added AN signal on top of OFDM data signal in such a way that when the AN passes through the channel it gets accumulated in Cyclic Prefix (CP) at the legitimate receiver only. Thus, it causes no interference at the legitimate receiver but degrades the performance of Eve. In [15], signal feature suppression based PLS technique was proposed. In this technique certain signal features are suppressed to avoid eavesdropping, such as CP periodicity feature concealing. Furthermore, adaptation based security techniques are also very popular PLS techniques in which transmitter parameters are adjusted in order to fulfill the quality of service (QoS) requirement of the legitimate receiver only, for example, adaptive modulation and coding with Automatic Repeat Request (ARQ) [16], fading based subcarrier activation technique [17], optimal power allocation based techniques [18], channel shortening [19], OFDM-subcarrier index selection for enhancing PLS [20], etc. It may be noted that adaptation based techniques, such as adaptive modulation and coding, can jointly enhance the security and spectral efficiency of wireless systems [18].

Now moving from PLS techniques for OFDM to PLS for IM, there are a few interesting PLS techniques proposed in the literature for SM in MIMO systems [21-25]. In [21], authors proposed transmit precoding based PLS techniques for SM. Moreover, jamming signal based PLS techniques are presented in [22]. In [23], authors proposed PLS techniques based on exploiting the channel reciprocity of Time Division Duplex (TDD) system to redefine the transmit antenna indices. However, the proposed technique cannot secure data symbol modulation. In order to solve this deficiency, the authors in [24] proposed a technique in which the rotation of both the indices of transmit antennas and constellation symbols based on the channel state information of the legitimate receiver are exploited, thus, securing both Index Modulation and data symbol modulation. To the best of the authors' knowledge, the first work related to PLS in OFDM-IM has recently been introduced in [25]. The authors investigate the randomized mapping rules based on channel reciprocity in TDD mode in order to secure both data symbol modulation and Index Modulation but in that work spectral efficiency is not taken into account.

In the literature, the majority of the works related to PLS are focused on the enhancement of security, but only a few works are reported to focus on the joint consideration of both spectral efficiency and security. Moreover, there are some techniques in which security is achieved at the cost of loss in resources.

Inspired by the need for joint consideration of security and SE, in this paper, we propose algorithms for the enhancement of PLS of OFDM-IM and for the quality of service (QoS) based communication in order to enhance SE of OFDM-IM. The proposed algorithms are based on adaptive subcarrier switching and adaptive modulation. More specifically, three approaches are proposed, namely, OFDM with Adaptive Index Modulation and Fixed Constellation Modulation (OFDM-AIM-FCM) for enhancing PLS and SE, OFDM with Adaptive Index Modulation, and Adaptive Constellation Modulation (OFDM-AIM-ACM) for enhancing PLS and SE and OFDM with Variable Index Modulation and Variable Constellation Modulation (OFDM-VIM-VCM) for QoS based communication in order to enhance SE. In particular, the first two approaches are based on channel based adaptation of subcarrier activation ratios and Constellation Modulation orders of subblocks in OFDM-IM by utilizing channel reciprocity concept in TDD mode while the third approach is based on QoS based adaptation. The works in $[8,9]$ focus on spectral efficiency alone without considering security concerns while first two proposed schemes provide security with some enhancement in spectral efficiency. The scheme in [9] and our third proposed algorithm both target enhanced SE. However, our proposed technique keeps the benefits of OFDM-IM in terms of low ICI and high EE, whereas the scheme presented in [9] does not keep these benefits.

The rest of the paper is organized as follows. The basic system model is presented in Section 2. The details of basic adaptive OFDM-IM are described in Section 3.1. The details of the developed algorithms are revealed in Section 3.2. The throughput of the proposed algorithms is presented in Section 4.1 while the theoretical BER performance analysis of the adaptive OFDM-IM (OFDM-ACM-FIM) is discussed in Section 4.2. Computer simulation results are exhibited and discussed in Section 5. Finally, the paper is concluded in Section 6.

Bold, lowercase, and capital letters are used for column vectors and matrices, respectively. $\operatorname{rank}(\mathbf{A})$ and $\operatorname{det}(\mathbf{A})$ denote the rank and determinant of $\mathbf{A}$, respectively. $\lambda_{i}(A)$ is the $i_{\text {th }}$ eigenvalue of $\mathbf{A}$. The expectation of an event is denoted by $E\{\cdot\}$ and $P(\cdot)$ stands for probability of an event. $\mathcal{S}$ denotes the complex signal constellation of size $M .\lfloor\cdot\rfloor$ is the floor function and $Q(\cdot)$ denotes the tail probability of the standard Gaussian distribution. $\mathscr{C} \mathscr{N}\left(0, \sigma_{X}^{2}\right)$ represents the distribution of a circularly symmetric complex Gaussian random variable $X$ with variance $\sigma_{X}^{2} \cdot(\cdot)^{H}$ and $(\cdot)^{T}$ denote Hermitian transposition and transposition, respectively.

\section{System Model and Preliminaries}

In this work, we consider a Single Input Single Output (SISO) OFDM-IM system. The basic system model consists of a legitimate transmitter ( $\mathrm{Tx}$ ), Alice, that wants to communicate securely with a legitimate receiver $(\mathrm{Rx}), \mathrm{Bob}$, in the presence of an illegitimate node, Eve, as shown in Figure 1, where TDD is considered as an operational mode. The notations $\mathbf{h}_{a b}\left(\mathbf{h}_{b}\right) \epsilon^{[1 \times L]}$ and $\mathbf{h}_{a e}\left(\mathbf{h}_{e}\right) \epsilon^{[1 \times L]}$ denote the slow varying multipath Rayleigh fading exponentially decaying channel from Alice to Bob and Alice to Eve, respectively, where $L$ is the length of the channel. In this work, Eve is considered to be passive, and hence there is no knowledge of Eve's channel at Alice. Moreover, it is also assumed that Eve is not very 


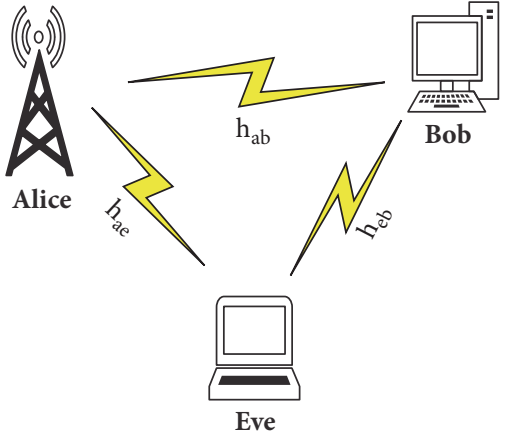

FIGURE 1: System model.

close to Bob such that Bob and Eve will have independent channel realizations [20]. In addition, the property of channel reciprocity is also adopted in this work, where the channel from Alice to Bob $\left(\mathbf{h}_{a b}\right)$ can be estimated from the channel of Bob to Alice $\left(\mathbf{h}_{b a}\right)$ in TDD.

\section{Adaptive OFDM-IM Model and Proposed Algorithms}

In this Section, basic concepts related to OFDM-IM with respect to adaptivity as well as proposed algorithms for enhancing PLS and SE are presented.

3.1. Adaptive OFDM-IM Model. In this subsection, OFDMIM model [5, 6] with respect to channel based adaptation (CBA) is explained. In this system, we employ a simplified OFDM-IM model as presented in Figures 2 and 3, where Figure 2 presents the OFDM-IM transmitter (Tx) while Figure 3 presents the OFDM-IM receiver $(\mathrm{Rx})$, respectively. Let us suppose that $m_{i}$ number of information bits, corresponding to $i_{t h}$ block, enters the OFDM-IM for the transmission, where the value of $m_{i}$ is different for different OFDM-IM blocks due to CBA and will be explained in Section 3.2. These $m_{i}$ bits are split into $G$ groups, such that each group contains $p_{j}$ bits, where $j \in\{1, \ldots, G\}$. The $p_{j}$ may be different for different groups based on CBA. The total number of bits in $i_{\text {th }}$ block can be represented as follows:

$$
m_{i}=\sum_{j=1}^{G} p_{j}
$$

In OFDM-IM, the subcarriers are also divided into $G$ subblocks. The number of subcarriers in any subblock, $\beta$, is $n$, where $n=N / G$ and $N$ is the total number of subcarriers in any OFDM-IM block. After that, $p_{j}$ bits of each group are mapped to corresponding subblock, $\beta$. This mapping is done by means of symbols and by the indices of subcarriers based on CBA.

The $p_{j}$ bits of each group are divided into $p_{1 j}$ and $p_{2 j}$ bits, where $p_{1 j}$ bits are carried by indices and $p_{2 j}$ bits are carried by symbols. More specifically, in each OFDM subblock, $k_{j}$ out of $n$ subcarriers are active and selected by index
TABLE 1: Lookup table for SAR values of $\{1 / 4,2 / 4,3 / 4\}$.

(a) SAR: $[1 / 4]$

\begin{tabular}{lc}
\hline Bits & Subcarrier indices \\
\hline 00 & 1 \\
\hline 01 & 2 \\
\hline 10 & 3 \\
\hline 11 & 4 \\
\hline
\end{tabular}

(b) SAR: $[2 / 4]$

\begin{tabular}{lc}
\hline Bits & Subcarrier indices \\
\hline 00 & 1,2 \\
\hline 01 & 2,3 \\
\hline 10 & 3,4 \\
\hline 11 & 1,4 \\
\hline
\end{tabular}

(c) SAR: $[3 / 4]$

\begin{tabular}{lc}
\hline Bits & Subcarrier indices \\
\hline 00 & $1,2,3$ \\
\hline 01 & $1,2,4$ \\
\hline 10 & $1,3,4$ \\
\hline 11 & $2,3,4$ \\
\hline
\end{tabular}

selector based on $p_{1 j}$ bits while the symbols corresponding to inactive subcarriers are set to zero. In the proposed work, each subblock may have different Subcarrier Activation Ratio (SAR), $k_{j} / n$, and Constellation Modulation (CM) order based on CBA. In this work, we consider four cases for SAR that are $1 / 4,2 / 4,3 / 4$, and $4 / 4$ and four cases of CM that are 2, 4, 8, and 16. The index selector of OFDM-IM uses a predefined lookup table for each subblock based on its SAR. Table 1 presents lookup tables for SARs of $1 / 4,2 / 4$, and $3 / 4$, while the case of SAR value of $4 / 4$ does not require any lookup table because no information is sent by indices (Classical OFDM). The remaining $p_{2 j}$ bits are mapped on to $\mathrm{M}$-ary data symbols, based on subblock CM, that modulates the active subcarriers. In this way, the information is conveyed by both indices of subcarriers and M-ary symbols that modulate the active subcarriers.

The selected indices are given by $I_{\beta}=\left\{i_{\beta, 1}, \ldots, i_{\beta, k_{j}}\right\}$, where $\beta \in\{1, \ldots, G\}, i_{\beta, \gamma} \in\{1, \ldots, n\}$, and $\gamma \in\left\{1, \ldots, k_{j}\right\}$. Therefore, the total number of bits carried by the indices of all $G$ groups in the $i_{t h}$ block is given by

$$
\begin{aligned}
& m_{1 i}=\sum_{j=1}^{G} p_{1 j}, \\
& p_{1 j}=\left\lfloor\log _{2}\left(\begin{array}{l}
n \\
k_{j}
\end{array}\right)\right\rfloor .
\end{aligned}
$$




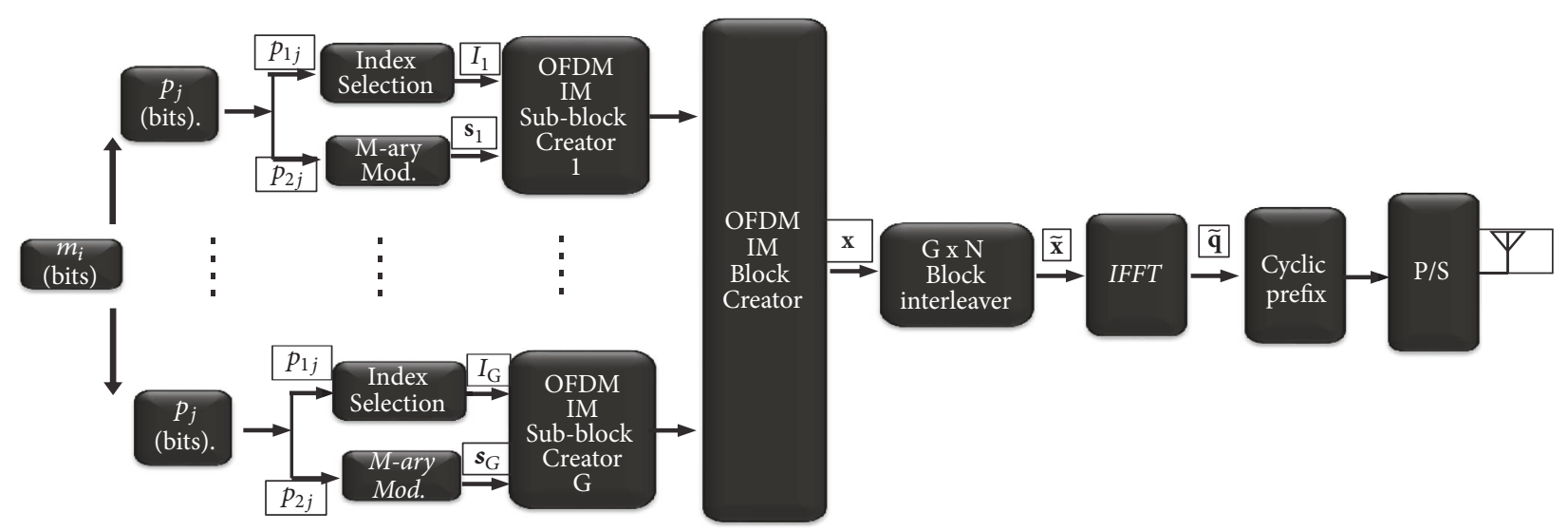

Figure 2: Basic OFDM-IM Tx.

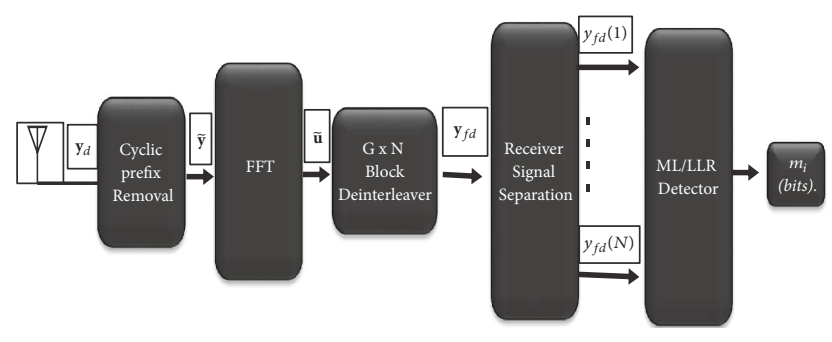

Figure 3: Basic OFDM-IM Rx.

Hence, $I_{\beta}$ has $c=2^{p_{1 j}}$ possible realizations. On the other hand, the total number of information bits carried by $\mathrm{M}$-ary signal constellations is given by

$$
\begin{aligned}
& m_{2 i}=\sum_{j=1}^{G} p_{2 j}, \\
& p_{2 j}=k_{j} \log _{2} M_{j},
\end{aligned}
$$

where $M_{j}$ is the modulation order and $k_{j}$ is the number of active subcarriers in each subblock. In this scheme, the total number of active subcarriers in each OFDM block is given as $K=\sum_{j=1}^{G} k_{j}$. The output of M-ary modulator is given as

$$
\mathbf{s}_{\beta}=\left[s_{\beta}(1), \ldots, s_{\beta}\left(k_{j}\right)\right] \text {, }
$$

where $s_{\beta}(\gamma) \in \mathcal{S}$. It should also be noted that the signal constellation is normalized to have unit average power. Finally, the OFDM block creator uses $I_{\beta}$ and $\mathbf{s}_{\beta}$ to create all of subblocks and then forms $N \times 1$ main OFDM-IM block by concatenation of $G$ subblocks and is given by

$$
\mathbf{x}=\left[x_{1}, x_{2}, \ldots, x_{N}\right]^{T} \text {. }
$$

where $x(\alpha) \in\{0, \delta\}, \alpha \in\{1, \ldots, N\}$. After this point, the block $\mathbf{x}$ is passed through $G \times N$ interleaver to ensure that the subcarriers in each subblocks are affected by uncorrelated wireless fading channels.

The resultant OFDM block after interleaver, $\widetilde{\mathbf{x}}$, is then passed through IFFT process, $(N / \sqrt{K}) \operatorname{IFFT}\{\widetilde{\mathbf{x}}\}$, which maps the frequency domain data symbols to time domain points represented as follows:

$$
\widetilde{\mathbf{q}}=\left[q_{1}, q_{2}, \ldots, q_{N}\right]^{T}
$$

In order to avoid ISI, a CP of length $\left(L_{c p}\right)$ is added at the beginning of each block, where $L_{c p}$ is assumed to be equal to or greater than the channel delay spread. Finally, the resultant signal $\widetilde{\mathbf{q}} \in C^{[N+L \times 1]}$ is transmitted through the Rayleigh fading channel, which is assumed to be constant during the transmission of each OFDM block and reaches both Bob and Eve. The baseband signal received at Bob can be represented as

$$
\mathbf{y}_{b}=\mathbf{h}_{b} * \widetilde{\mathbf{q}}+\mathbf{z}_{b}
$$

where $\mathbf{h}_{b}$ is the channel impulse response seen by Bob and $\mathbf{z}_{b}$ represents additive white Gaussian noise (AWGN) at Bob with distribution of $\mathscr{C} \mathscr{N}\left(0, N_{0, T}\right)$. Similarly, the baseband signal received at Eve is given by

$$
\mathbf{y}_{e}=\mathbf{h}_{e} * \widetilde{\mathbf{q}}+\mathbf{z}_{e}
$$

where $\mathbf{h}_{e}$ is the channel impulse response seen by Eve and $\mathbf{z}_{e}$ represents AWGN at Eve with distribution of $\mathscr{C} \mathscr{N}\left(0, N_{0, T E}\right)$.

The basic block diagram of the receiver is presented in Figure 3. The receiver (both Bob and Eve) first removes the $\mathrm{CP}$ and then applies FFT on the received time domain signal $\mathbf{y}_{d}$ with normalization factor of $K / \sqrt{N}$ and finally deinterleaves the resultant signal to get the received signal, $\mathbf{y}_{f d}=\left[y_{f d}(1), y_{f d}(2), \ldots, y_{f d}(N)\right]^{T}$, in frequency domain, where $d$ can be Bob or Eve.

The receiver task is to detect the indices of active subcarriers and corresponding symbols by processing, $y_{f d}(\alpha)$, where $\alpha=\{1, \ldots, N\}$. In our algorithm, we use lookup table based modified Log-likelihood-Ratio (LLR) detector for detection of active indices for each subblock [6]. First of all, LLR values of frequency domain symbols corresponding to each subcarrier are calculated as follows:

$$
\lambda(\alpha)=\ln \left(\frac{\sum_{\chi=1}^{M} P\left(x(\alpha)=s_{\chi} \mid y_{f d}(\alpha)\right)}{P\left(x(\alpha)=0 \mid y_{f d}(\alpha)\right)}\right)
$$


The above equation can be further simplified by using Bayes' formula as

$$
\begin{aligned}
& \lambda(\alpha) \\
& =\ln (k)-\ln (n-k)+\frac{\left|y_{f d}(\alpha)\right|^{2}}{N_{0, f}} \\
& \quad+\ln \left(\sum_{\chi=1}^{M} \exp \left(-\frac{1}{N_{0, f}}\left|y_{f d}(\alpha)-h_{F}(\alpha) s_{\chi}\right|^{2}\right)\right)
\end{aligned}
$$

where $N_{0, f}$ is the noise variance in frequency domain $\left(N_{0, f}=\right.$ $\left.(K / N) N_{0, T}\right)$. In (12), numerical overflow can be prevented by using the Jacobian logarithm [27]. For example, for $M=2$ and $k_{j}=n / 2$, (12) simplifies to

$$
\begin{aligned}
\lambda(\alpha)= & \max (a, b)+\ln (1+\exp (-|b-a|)) \\
& +\frac{\left|y_{f d}(\alpha)\right|^{2}}{N_{0, f}}
\end{aligned}
$$

where $b=-\left|y_{f d}(\alpha)+h_{f}(\alpha)\right|^{2} / N_{0, f}$ and $a=-\mid y_{f d}(\alpha)-$ $\left.h_{f}(\alpha)\right|^{2} / N_{0, f}$. In our work, we also use higher order modulation and use the following identity: $\ln \left(e^{a_{1}}+e^{a_{1}}+\right.$ $\left.\cdots+e^{a_{M}}\right)=\left(f_{\text {max }}\left(f_{\max }\left(\ldots f_{\max }\left(f_{\max }\left(a_{1}, a_{2}\right), a_{3}\right), \ldots\right), a_{M}\right)\right)$, where $f_{\max }(a, b)=\ln \left(e^{a_{1}}+e^{a_{1}}\right)=\max \left(a_{1}, a_{2}\right)+\ln \left(1+e^{-\left|a_{1}-a_{2}\right|}\right)$.

In order to detect the active indices, LLR value corresponding to each subcarrier is calculated using (12). Afterwards, the receiver calculates the sum of LLRs corresponding to each combination of the subcarriers in the lookup table with respect to subblock based SAR as follows:

$$
d_{\beta}^{w}=\sum_{\gamma=1}^{k_{j}} \lambda\left(n(\beta-1)+i_{\beta, \gamma}^{w}\right)
$$

where $w=1, \ldots, c$ and $c$ is the total number of combinations of subcarriers in the lookup table with respect to any SAR. The receiver makes a decision of set of active indices by selecting the set with maximum value of sum of LLRs as follows:

$$
\widehat{w}=\arg \max _{w} d_{\beta}^{w}
$$

After selecting the set with maximum LLR, the receiver gets the set of active indices corresponding to SAR. After the detection of active subcarrier, the information is then passed to index demapper based on lookup table to estimate $m_{1 i}$ bits. After determination of active indices, the demodulation of the constellation symbols ( $\mathrm{M}$-ary symbols) is carried out and finally we get $m_{2 i}$ bits.

3.2. Proposed Algorithms for OFDM-IM. In this subsection, proposed algorithms for enhancing PLS and spectral efficiency are presented.

3.2.1. OFDM-AIM-FCM. In OFDM-AIM-FCM, SAR for each subblock is changed adaptively while fixed CM is used for all subblocks. The basic idea of OFDM-AIM-FCM is presented in Figure 4. The basic steps for OFDM-AIM-FCM algorithm are as follows:

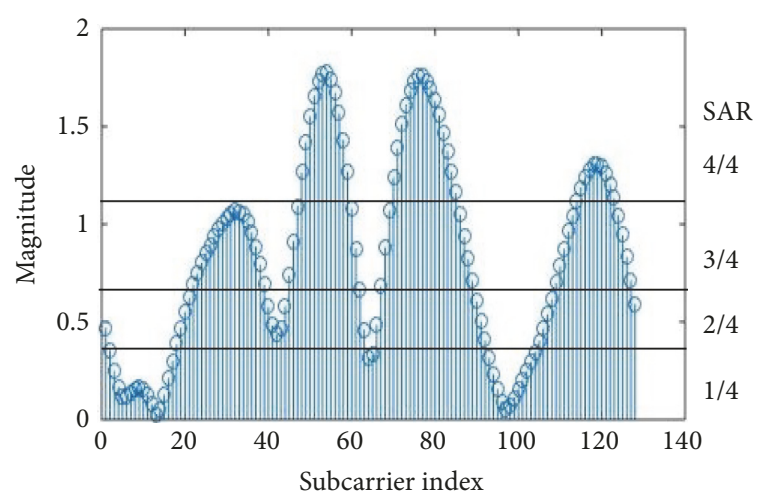

Figure 4: Proposed: OFDM-IM-AIM-FCM.

(i) In the first step, the channel is estimated at all nodes. In order to do that, Alice and Bob send a reference signal to each other (within coherence time). After channel estimation, they take FFT to convert the channel coefficient vector into frequency domain vector, $\mathbf{h}_{f}$

(ii) Afterwards, the vector $\mathbf{h}_{f}$ at each node is divided into $G$ subblocks with $n$ elements in each of, $\beta$, subblock, where $n=N / G$

(iii) In the next step, the average, $a v(\beta)$, of absolute values of subblock's elements is calculated as follows:

$$
\operatorname{av}(\beta)=\frac{\sum_{r=1}^{n}\left|h s_{\beta, r}\right|}{n}
$$

where $h s_{\beta, r}$ is the $r_{t h}$ element of $\beta$ subblock

(iv) After finding the average value, $a v(\beta)$, for each of $G$ subblocks, they are divided into four groups based on their $a v(\beta)$. More specifically, find the mean, $m e$, of $\mathbf{a v}$, where av is a vector containing average values for all subblocks. Afterwards, divide the subblocks into two groups, $\mathbf{g}_{1}$ and $\mathbf{g}_{2}$, by comparing their corresponding $a v(\beta)$ values with me. The subgroup $\mathbf{g}_{1}$ contains those subblocks whose $a v(\beta)$ values are greater than or equal to me while $\mathbf{g}_{2}$ contains those subblocks whose values of $a v(\beta)$ are less than $m e$. Afterwards, both $\mathbf{g}_{1}$ and $\mathbf{g}_{2}$ are further divided into two subgroups by using mean method as explained above. As a result, $G$ subblocks are divided into four groups such as $\mathbf{g}_{11}, \mathbf{g}_{22}, \mathbf{g}_{33}$, and $\mathbf{g}_{44}$. The resultant groups are sorted in descending order in terms of average channel magnitude such that $\mathbf{g}_{11}$ contains those subblocks that have the highest values of $a v(\beta)$ while $\mathbf{g}_{44}$ contains subblocks with the lowest values of $\operatorname{av}(\beta)$

(v) Finally, higher SAR values are selected for those groups that have higher values of $a v(\beta)$ while lower values of SAR are selected for those groups that have lower values of $a v(\beta)$, such that SAR values of $4 / 4$, $3 / 4,2 / 4$, and $1 / 4$ are selected for groups $\mathbf{g}_{11}, \mathbf{g}_{22}, \mathbf{g}_{33}$, and $\mathbf{g}_{44}$, respectively, as presented in Table 2 
TABLE 2: OFDM-AIM-FCM.

\begin{tabular}{lc}
\hline Group & SAR \\
\hline $\mathbf{g}_{11}$ & $4 / 4$ \\
\hline $\mathbf{g}_{22}$ & $3 / 4$ \\
\hline $\mathbf{g}_{33}$ & $2 / 4$ \\
\hline $\mathbf{g}_{44}$ & $1 / 4$ \\
\hline
\end{tabular}

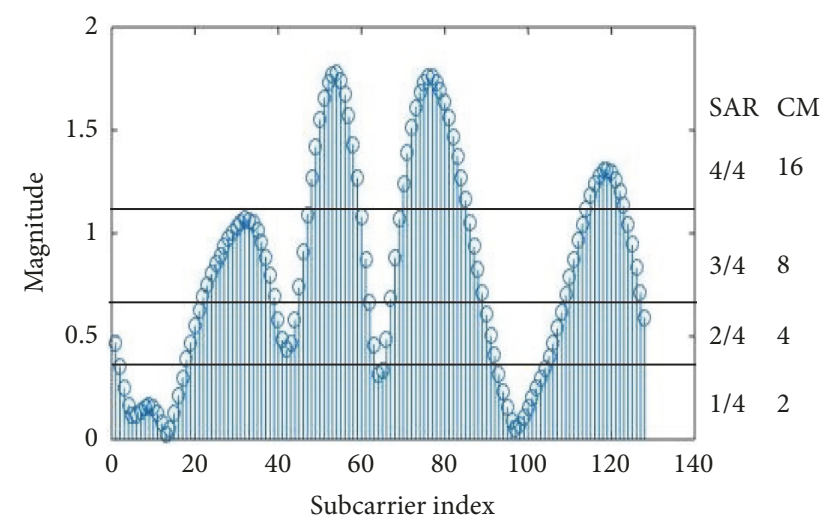

Figure 5: Proposed: OFDM-AIM-ACM.

Based on the above algorithm (OFDM-AIM-FCM), Alice determines SAR for each subblock and the total number of bits, $m_{i}$, for $i_{t h}$ block. Afterwards, data is loaded to the indices and the symbols based on adaptive SAR and fixed CM and a block is generated using adaptive OFDM-IM model explained in Section 3.1. Finally, the resultant signal $\widetilde{\mathbf{q}} \in$ $C^{[N+L \times 1]}$ is transmitted through the Rayleigh fading channel and reaches both Bob and Eve.

Bob and Eve will then detect the active subcarriers based on SAR values of subblocks with respect to OFDMAIM-FCM. The resultant information is passed to the index demapper that provides the information carried by indices. After determination of active indices, constellation symbols are demodulated.

Thanks to channel decorrelation assumptions, Bob and Eve will have differences in their determined subblock based SAR values. Due to channel reciprocity employment, the SAR values for different subblocks determined by Bob are similar to that of Alice's while they are different at Eve. This dissimilarity in SAR values for different subblocks at Eve leads to wrong detection of bits at Eve. Hence, there is a performance gap at Bob and Eve, which enables the secure communication between Alice and Bob.

3.2.2. OFDM-AIM-ACM. In OFDM-AIM-ACM both the SAR and CM order are adaptively varied based on channel of legitimate node in order to enhance PLS and SE. The basic concept of OFDM-AIM-ACM is presented in Figure 5.

(i) First four steps of OFDM-AIM-ACM are similar to that of OFDM-AIM-FCM. Specifically, $\mathbf{h}_{f}$ vector is divided into $G$ subblocks. The resultant $G$ subblocks are grouped into four groups such as $\mathbf{g}_{11}, \mathbf{g}_{22}, \mathbf{g}_{33}$, and $\mathbf{g}_{44}$ using mean method as explained earlier
TABLE 3: OFDM-AIM-ACM.

\begin{tabular}{lcc}
\hline Group & SAR & $M$ \\
\hline $\mathbf{g}_{11}$ & $4 / 4$ & 16 \\
\hline $\mathbf{g}_{22}$ & $3 / 4$ & 8 \\
\hline $\mathbf{g}_{33}$ & $2 / 4$ & 4 \\
\hline $\mathbf{g}_{44}$ & $1 / 4$ & 2 \\
\hline
\end{tabular}

(ii) The basic difference in OFDM-AIM-ACM as compared to OFDM-AIM-FCM is that both the SAR and $\mathrm{CM}$ order are varied adaptively for each subblock in it. In OFDM-AIM-ACM, higher SAR with higher order $\mathrm{CM}$ are selected for those groups that have high values of $a v(\beta)$ while lower values of SAR with lower order $\mathrm{CM}$ are selected for those groups that have lower values of $a v(\beta)$. Based on OFDM-AIM-ACM, SAR value of $4 / 4$ is selected with $M=16,3 / 4$ with $8,2 / 4$ with 4 , and $1 / 4$ with 2 for groups $\mathbf{g}_{11}, \mathbf{g}_{22}, \mathbf{g}_{33}$, and $\mathbf{g}_{44}$, respectively, as presented in Table 3

Based on the above-mentioned algorithm (OFDM-AIMACM), Alice determines SAR and CM order for each subblock and the total number of bits, $m_{i}$, for $i_{t h}$ block. Afterwards, data is loaded to the indices and symbols based on adaptive SAR and adaptive CM and finally a block is generated using adaptive OFDM-IM model explained in Section 3.1. Finally, the resultant signal $\widetilde{\mathbf{q}} \in C^{[N+L \times 1]}$ is transmitted through the Rayleigh fading channel and reaches both Bob and Eve.

Bob and Eve will first detect active subcarriers based on subblock-SAR values with respect to OFDM-AIM-ACM. The resultant information is then passed to the index demapper which provides the information carried by indices. After determination of active indices, constellation symbols are demodulated based on subblock CM order with respect to OFDM-AIM-ACM.

Due to the channel decorrelation, the SAR values and CM orders of different subblocks determined by Bob and Eve based on OFDM-AIM-ACM are different. The SAR values and $\mathrm{CM}$ orders of different subblock determined by Bob are similar to that of Alice's due to channel reciprocity, while it is different at Eve as compared to Alice. This difference in subblock based SAR values as well as CM order at Eve compared to Alice will cause errors in the detection of data carried by indices and symbols. Hence, there is a significant performance gap between Bob and Eve. This performance gap will ensure secure communication between Alice and Bob. It should also be noted that OFDM-AIM-ACM is more difficult to be attacked as compared to OFDM-AIM-FCM because in the latter case only subblock based SAR is varied adaptively, while in the former both the SAR and CM are varied adaptively.

3.2.3. OFDM-VIM-VCM for QoS. In OFDM-VIM-VCM, the IM and CM order are varied for QoS based communication in order to maximize the spectral efficiency. The basic motivation behind this approach is that, instead of using complex 
optimization based approaches for maximizing spectral efficiency, simple simulation based approach is proposed for this purpose. The basic concept is to vary the SAR and CM with the change in average SNR to maximize the spectral efficiency while fulfilling certain QoS requirement. The basic procedure can be summarized as follows:

(i) First, OFDM-IM is implemented with different modulation order for each SAR. Afterwards, BER and throughput curves are simulated for each of SAR value with higher order modulation; for example, in this work, we are considering SAR values of $1 / 4,2 / 4$, $3 / 4$, and $4 / 4$ and CM order of 2, 4, 8, and 16

(ii) Then, all BER curves are merged in one figure and all throughput curves in another figure

(iii) In the next step, certain BER curves are selected based on their performance gap and throughput values. More specifically, among the BER curves that have similar performance, select a curve that has maximum value of throughput. From the selected curves in the former step, select those curves that have a performance gap between them. Afterwards, the throughput curves corresponding to selected BER curves are also selected

(iv) Finally, switching table is constructed based on QoS requirement. The table depicts the values of different SAR and CM of system for different average SNR ranges to maximize the spectral efficiency while fulfilling QoS requirements

(v) After construction of switching table, this table is then used for QoS based communication for maximizing spectral efficiency

\section{Performance Analysis of Adaptive OFDM- IM Scheme}

4.1. Throughput of Adaptive OFDM-IM. This section presents the details related to the throughput of the adaptive OFDMIM. The throughput for adaptive OFDM-IM can be given as

$$
\text { Throughput }=\frac{\sum_{j=1}^{G} p_{1 j}+\sum_{j=1}^{G} p_{2 j}}{N+N_{C P}}
$$

where $p_{1 j}=\left\lfloor\log _{2}\left(\begin{array}{c}n \\ k_{j}\end{array}\right)\right\rfloor$ and $p_{2 j}=k_{j} \log _{2} M_{j}$. The basic difference between conventional OFDM-IM and adaptive OFDM-IM is in $k_{j}$ and $M_{j}$ which are fixed in the former but vary adaptively in the latter. In case of OFDM-ACMFCM, $k_{j}$ is different for different subblocks and $M_{j}$ is the same for all subblocks while in case of OFDM-ACM-AIM and OFDM-VIM-VCM both $k_{j}$ and $M_{j}$ are different for different subblocks.

\subsection{Performance Analysis of Adaptive OFDM-IM Scheme.} This section presents the analytical evaluation for the upper bound of the average bit error probability (ABEP) of the adaptive OFDM-IM scheme (OFDM-AIM-FCM with $M=$ 2) based on pairwise error probability (PEP). In this analysis,
ML detector with a lookup table is considered whose results are equal to and applicable to the modified LLR detector (near ML) with a lookup table. This is because of the fact that the error performance of ML detector is almost similar to that of modified LLR detector as explained in [6].

In the conventional OFDM-IM, the same SAR values are used in all subblocks while in case of OFDM-AIM-FCM different SARs values are used in different subblocks. As explained earlier, there are $N$ subcarriers that are divided into $G$ subblocks with $n$ subcarriers in each subblock. In OFDMAIM-FCM, the subblocks are divided into four groups, $\mathbf{g}_{44}$, $\mathbf{g}_{33}, \mathbf{g}_{22}$, and $\mathbf{g}_{11}$, with SAR values of $1 / 4,2 / 4,3 / 4$, and $4 / 4$, respectively, used in them. In order to simplify the analysis, we can assume that the size of each of the above-mentioned groups in OFDM-AIM-FCM is the same. It should be noted that the PEP event is similar in the subblocks corresponding to the same group and is different for subblocks that belong to different groups.

In the first step, the average bit error probability (ABEP) of first subblock of first group is calculated and then the results are extended to include subblocks of other groups. Afterwards, we will find average ABEP for each group and finally find the ABEP of adaptive OFDM-IM subblock.

The input-output relationship in frequency domain for the first subblock of first group is given as follows:

$$
\mathbf{y}=\mathbf{X h}+\mathbf{w}
$$

where $\mathbf{X}$ is an $n \times n$ diagonal matrix containing $[x(1), x(2), \ldots, x(n)]^{T}$ as diagonal data elements, $\mathbf{y}$ is the received signal subvector containing $\left[y_{f d}(1), y_{f d}(2), \ldots, y_{f d}(n)\right]^{T}, \mathbf{h}$ is the channel subvector containing $\left[h_{f}(1), h_{f}(2), \ldots, h_{f}(n)\right]^{T}$, and $\mathbf{w}$ is the noise subvector containing $[w(1), w(2), \ldots, w(n)]^{T}$. Let us assume that $\mathbf{K}_{\mathbf{n}}=E\left[\mathbf{h} \mathbf{h}^{H}\right]$ is a covariance submatrix of rank $r_{1}$ $\left(r_{1}=\operatorname{rank}\left(\mathbf{K}_{\mathbf{n}}\right)\right)$. This matrix is valid for all subblocks. Moreover, the concatenation of these small covariance submatrices gives $\mathbf{K}$ matrix which is the covariance matrix of $\mathbf{h}_{f}$.

Let us suppose that $\mathbf{X}$ signal is transmitted through channel and received as erroneous signal $\widehat{\mathbf{X}}$. The receiver can make decision error in both constellation symbols and indices. One of the best ways to analyse these errors is in terms of PEP. In [28], an expression for conditional pairwise error probability (CPEP) is presented for the model of (18) and is given as

$$
P(\mathbf{X} \longrightarrow \widehat{\mathbf{X}} \mid \mathbf{h})=Q\left(\sqrt{\frac{\delta}{2 N_{0, f}}}\right),
$$

where $\delta=\mathbf{h}^{\mathbf{H}} \mathbf{A h}$ and the $\mathbf{A}$ matrix equals to $(\mathbf{X}-\widehat{\mathbf{X}})^{H}(\mathbf{X}-\widehat{\mathbf{X}})$. In order to find the unconditional pairwise error probability (UPEP), the expectation of CPEP is taken with respect to the channel and is given as follows: $P(\mathbf{X} \longrightarrow \widehat{\mathbf{X}})=E_{h}\{Q(x)\}$. Based on [29], we can define an orthogonal matrix $\mathbf{F}$ where $\mathbf{F}^{\mathbf{H}} \mathbf{F}=\mathbf{I}$. The covariance submatrix and channel can be simplified as $\mathbf{K}_{\mathbf{n}}=\mathbf{F D F}^{\mathbf{H}}$ and $\mathbf{h}=\mathbf{F u}$, respectively. Here, $\mathbf{D}$ is a diagonal matrix and is equal to $\mathbf{D}=E\left[\mathbf{u} \mathbf{u}^{\mathbf{H}}\right]=\mathbf{D}$ and $\mathbf{u}$ 
is eigen vector. Using the probability density function (p.d.f.) of $\mathbf{u}$ [6] and simplification of $Q(x)$ and $\delta$, the unconditional pairwise error probability (UPEP) can be written as

$$
P(\mathbf{X} \longrightarrow \widehat{\mathbf{X}})=\frac{1 / 12}{\operatorname{det}\left(\mathbf{I}_{\mathbf{n}}+q_{1} \mathbf{B}\right)}+\frac{1 / 4}{\operatorname{det}\left(\mathbf{I}_{\mathbf{n}}+q_{2} \mathbf{B}\right)},
$$

where $\mathbf{I}_{\mathbf{n}}$ is an identity matrix, $\mathbf{B}=\mathbf{A} \mathbf{K}_{\mathbf{n}}, q_{1}=1 /\left(4 N_{0, f}\right)$, and $q_{2}=1 /\left(3 N_{0, f}\right)$. The above equation can be further simplified as follows:

$$
\begin{aligned}
P(\mathbf{X} \longrightarrow \widehat{\mathbf{X}})= & \left(12 q_{1}^{r} \prod_{\xi=1}^{r} \lambda_{\xi}(B)\right)^{-1} \\
& +\left(4 q_{2}^{r} \prod_{\xi=1}^{r} \lambda_{\xi}(B)\right)^{-1}
\end{aligned}
$$

where $r \leq \min \left\{r_{1}, r_{2}\right\}$ and $r_{2}=\operatorname{rank}(A)$. For different SAR, $r_{2}$ will be different, so (21) is still applicable to any SAR.

The overall average bit error probability of $\tau_{t h}$ subblock of any group can be calculated by using UPEP as follows:

$$
P_{b}^{\tau}(E) \approx \frac{1}{p^{\tau} n_{x}^{\tau}} \sum_{\mathbf{X}^{\tau}} \sum_{\widehat{\mathbf{X}}^{\tau}} P\left(\mathbf{X}^{\tau} \longrightarrow \widehat{\mathbf{X}}^{\tau}\right) \quad e\left(\mathbf{X}^{\tau}, \widehat{\mathbf{X}}^{\boldsymbol{\tau}}\right),
$$

where $p^{\tau}$ is the number of information bits in $\tau_{t h}$ subblock of any group, $n_{x}^{\tau}$ represents the number of realizations of $\mathbf{X}^{\tau}$, and $e\left(\mathbf{X}^{\boldsymbol{\tau}}, \widehat{\mathbf{X}}^{\boldsymbol{\tau}}\right)$ is the number of information bit errors committed by choosing $\widehat{\mathbf{X}}^{\boldsymbol{\tau}}$ instead of $\mathbf{X}^{\boldsymbol{\tau}}$. Using (22), the ABEP for $\Upsilon_{t h}$ group can be calculated as follows:

$$
P_{b}^{\Upsilon}(E) \approx \frac{1}{\digamma}\left(\sum_{\tau=1}^{F} P_{b}^{\tau}(E)\right)
$$

where $F$ is the number of subblocks in any group and $\digamma=8$ in our case. Equation (23) can be rewritten as follows:

$$
\begin{aligned}
& P_{b}^{\Upsilon}(E) \\
& \quad \approx \frac{1}{\digamma} \sum_{\tau=1}^{F}\left(\frac{1}{p^{\tau} n_{x}^{\tau}} \sum_{\mathbf{X}^{\tau}} \sum_{\widehat{\mathbf{X}}^{\tau}} P\left(\mathbf{X}^{\boldsymbol{\tau}} \longrightarrow \widehat{\mathbf{X}}^{\boldsymbol{\tau}}\right) e\left(\mathbf{X}^{\boldsymbol{\tau}}, \widehat{\mathbf{X}}^{\boldsymbol{\tau}}\right)\right)
\end{aligned}
$$

Finally, ABEP for the OFDM-IM block can be calculated as follows:

$$
P_{b}(E) \approx \frac{1}{\Omega} \sum_{\Upsilon=1}^{\Omega} P_{b}^{\Upsilon}(E) \approx \frac{1}{\Omega}\left(P_{b}^{1}+P_{b}^{2}+P_{b}^{3}+P_{b}^{4}\right)
$$

where $\Omega$ is the number of groups and in this case $\Omega=4$. The theoretical BER curve will be presented in Section 5 .

\section{Simulation Result}

This section presents the simulation results to evaluate the effectiveness of the proposed algorithms, named as OFDMAIM-FCM, OFDM-AIM-ACM, and OFDM-VIM-VCM by using bit error rate (BER) and throughput as performance metrics.
TABLE 4: System parameters.

\begin{tabular}{lc}
\hline Channel & Multipath Rayleigh fading channel \\
\hline Channel length & 10 \\
\hline OFDM frame size $(\mathrm{N})$ & 128 \\
\hline Length of subblock & 4 \\
\hline Detector & Modified LLR based detector \\
\hline
\end{tabular}

In this work, we consider an OFDM-IM system with $N=128$ subcarriers and a CP of length 10 . As explained in Section 3.1, OFDM-IM block is divided into $G=N / n=$ $128 / 4=32$ subblocks, where $n=4$ is the number of subcarriers in each subblock. The multipath Rayleigh fading channel is considered for both Bob and Eve with equal number of channel taps $(L=10)$. The basic simulation parameters are presented in Table 4 . In this work, lookup table based special LLR detector is employed at receiver, as explained in Section 3.1, to determine the active indices and corresponding constellation symbols based on the proposed algorithms. Additionally, we also consider that Eve knows our security algorithms. For simplicity and without loss of generality, CP is not considered in the throughput calculation.

It should be noted that the proposed scheme is a type of scheme which does not cause much difference in the SNR between Bob and Eve, but still Eve cannot decode, while Bob can decode (this case is somehow similar to the case of interleaver or precoder based security techniques [20,30]). In such cases, BER can be used as a metric to measure secrecy instead of secrecy capacity and secrecy outage probability as reported in $[20,30,31]$. Therefore, in this work, we use BER-based secrecy gap metric [20] to evaluate the secrecy. Furthermore, in this work we are targeting quality of service (QoS) based security $[16,32]$. The basic idea behind QoS based security is to secure different services (voice, video, etc.) instead of focusing on providing perfect secrecy. More specifically, it should be noted that perfect secrecy is not always needed to provide a perfectly secure service. In reality, each service has different QoS requirements than the others, and if we ensure that Eve is operating below these requirements, then practical secrecy can be guaranteed. So, in this work we target to provide security for services such as voice and video and make sure that error rate at Eve is greater than minimum required error rate criteria to use that service [16]. For example, voice and video can be made secure at Bob by making sure that PER (corresponding to BER) at Bob is less than minimum required PER (corresponding to BER) in order to use that service while PER at Eve is made greater than minimum required PER. The minimum $P E R$ requirement for different services is presented in Table 5 [26]. Hence, although the throughput is nonzero, the proposed scheme can still provide QoS based security (it should be noted that PER can be calculated from BER as follows: $\left.P E R=1-(1-B E R)^{n}\right)$, where $n$ is the block size [33]).

In the first phase, OFDM-IM is simulated for different SAR values, such as $1 / 4,2 / 4,3 / 4$, and $4 / 4$ based on lookup tables presented in Table 1 with FCM $(\mathrm{M}=2)$. Afterwards, we simulate OFDM-AIM-FCM for BPSK $(M=2)$ for PLS and 
TABLE 5: QoS lookup table [26].

\begin{tabular}{ll}
\hline Service & $P E R$ \\
\hline Voice & $10^{-2}$ \\
\hline Video & $10^{-3}$ \\
\hline
\end{tabular}

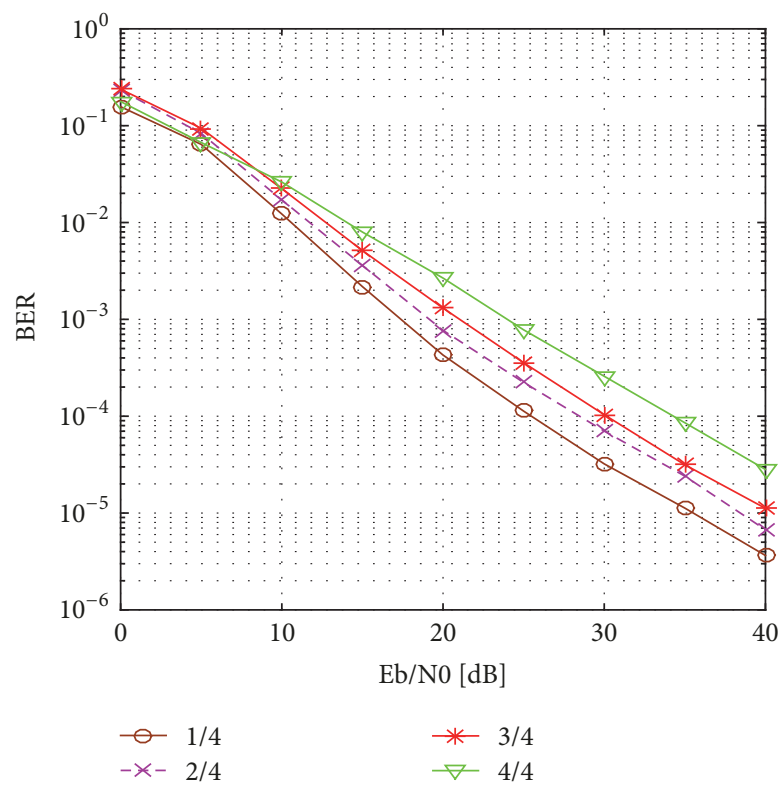

FIGURE 6: BER performance for OFDM-IM $(n=4, k=\{1,2,3,4\})$.

also extend it for higher order modulation such as $M=4$, $M=8$, and $M=16$. Then, OFDM-AIM-FCM is extended to OFDM-AIM-ACM for providing another stronger PLS technique. Finally, we implement OFDM-VIM-VCM for QoS based communication in order to maximize the spectral efficiency.

5.1. OFDM-AIM-FCM. Figure 6 presents the BER plots for OFDM-IM with different SAR values, such as $1 / 4,2 / 4,3 / 4$, and $4 / 4$ for $M=2$. It should be noted from Figure 6 that the BER performance for lower values of SAR is better than the case of higher values of SAR; for example, the BER performance of $1 / 4$ case is the best while BER performance of $4 / 4$ is the worst. The reason for the better performance of BER at lower SAR is due to the fact that in case of lower SAR there will be less noise in the frequency domain.

Figure 7 presents throughput for OFDM-IM with different SAR values for $M=2$. It should be noted that the throughput for the system improves as the activation ratio increases except for the case with SAR value of $3 / 4$ which outperforms $4 / 4$ case. The reason is that each subblock carries 4 bits in case of SAR value of $4 / 4$ while each block carries 5 bits in case of $3 / 4$.

Figure 8 presents a comparison of BER performances among the proposed OFDM-AIM-FCM scheme, the scheme presented in [24], and OFDM-IM $(n=4, k=2)$ (Ref. (Ref. means the reference scheme to which we compare our proposed algorithm)). It is observed from Figure 8 that the BER performances of OFDM-AIM-FCM and OFDM-IM [6]

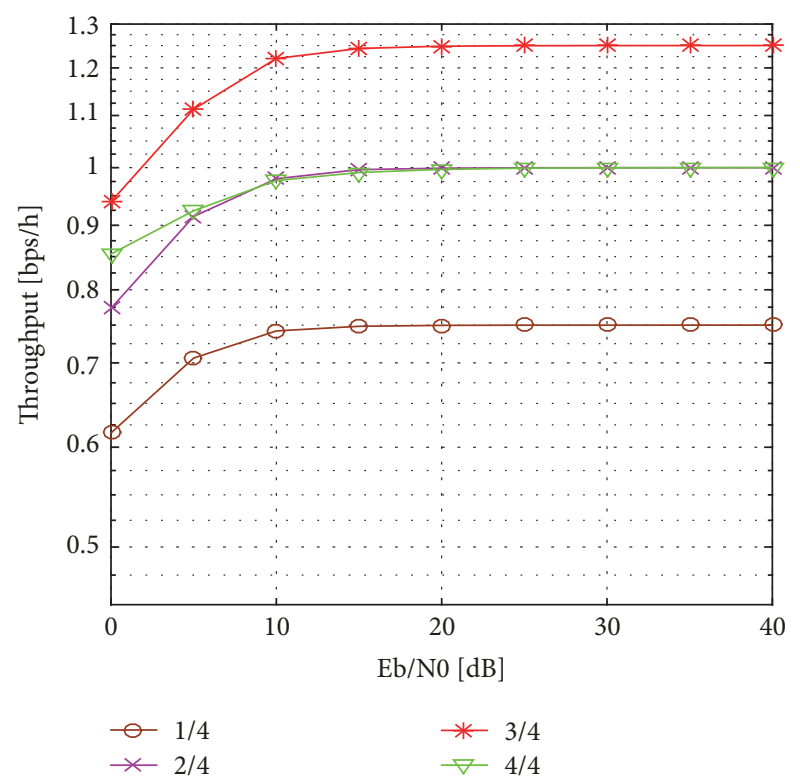

Figure 7: Throughput performance for OFDM-IM $(n=4, k=$ $\{1,2,3,4\})$.

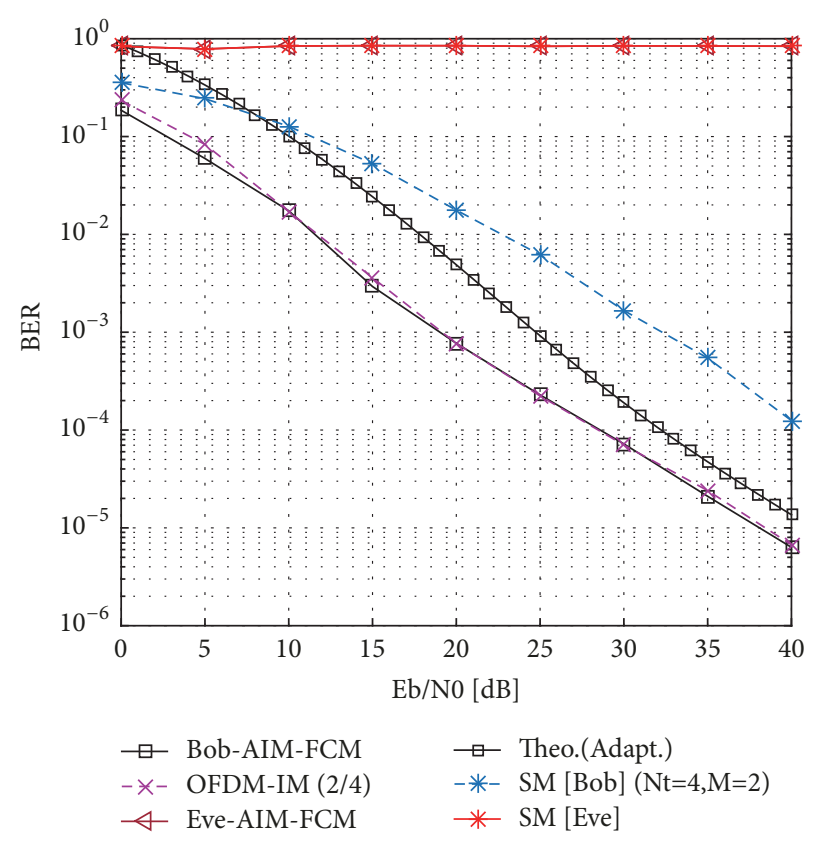

FIGURE 8: BER performance for OFDM-AIM-FCM, Secure SM [24] and OFDM-IM $(n=4, k=2)$.

are similar for the case of Bob but the scheme presented in [24] has the worst performance as compared to others. It is also observed that the performance of Eve is the worst for all values of SNR for the proposed OFDM-AIM-FCM technique and the scheme presented in [24] while her performance is similar to that of Bob for the cases of OFDMIM [6]. Hence, the proposed technique and the technique presented in [24] are secure as compared to OFDM-IM [6]. Figure 8 also presents the theoretical upper bound BER performance of OFDM-AIM-FCM based on (25). It should 


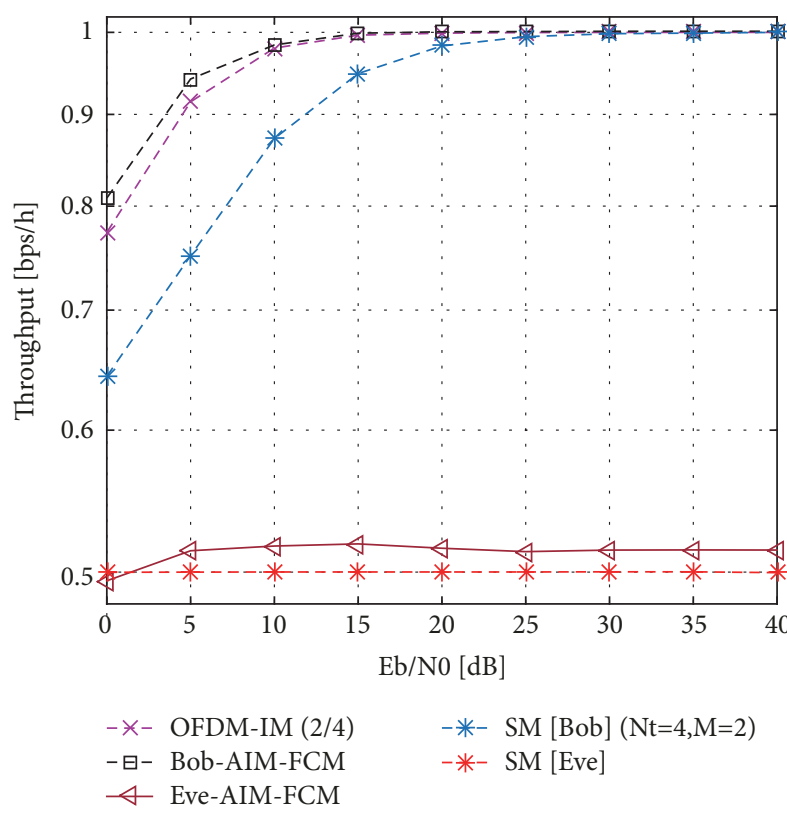

FIGURE 9: Throughput performance for OFDM-AIM-FCM, Secure SM [24] and OFDM-IM $(n=4, k=2)$.

be noted that theoretical curve becomes tight at higher SNR with the simulation curve.

Figure 9 shows the comparison of throughput performances among the proposed OFDM-AIM-FCM scheme, the scheme presented in [24], and OFDM-IM $(n=4, k=2)$ [6] with $M=2$. It is observed that the throughput performances of all of these schemes for Bob are approximately similar at higher values of SNR. At equivalent BER we can notice that the throughput of the proposed OFDM-AIM-FCM scheme outperforms the OFDM-IM (2/4) [6] at lower values of SNR. Moreover, the proposed scheme (OFDM-AIM-FCM) also outperforms in terms of throughput as compared to the scheme presented [24] at lower values of SNR. It is also observed that the throughput performance of Eve is the worst for the proposed OFDM-AIM-FCM technique and the scheme presented in [24] while her performance is similar to that of Bob for the case of OFDM-IM [6] scheme.

5.2. OFDM-AIM-ACM. Figure 10 presents the BER performance of Bob and Eve for OFDM-AIM-FCM for $M=2$, $M=4, M=8$, and $M=16$. It should be noted from the figure that as the modulation order increases the BER performance degrades. The performance of Eve for OFDMAIM-FCM is the worst for all cases of CM such as $M=2$, $M=4, M=8$, and $M=16$. Figure 10 also presents the BER performance of Bob and Eve for the proposed OFDM-AIMACM. It is observed from Figure 10 that the BER performance of OFDM-AIM-AIM is approximately the same as the case of OFDM-AIM-FCM for $M=8$, while the BER performance of Eve is the worst for all values of SNR. Hence, OFDMAIM-ACM can provide secure communication between Alice and Bob. Figure 11 presents throughput performance of Bob and Eve for OFDM-AIM-FCM with $M=2, M=4$,

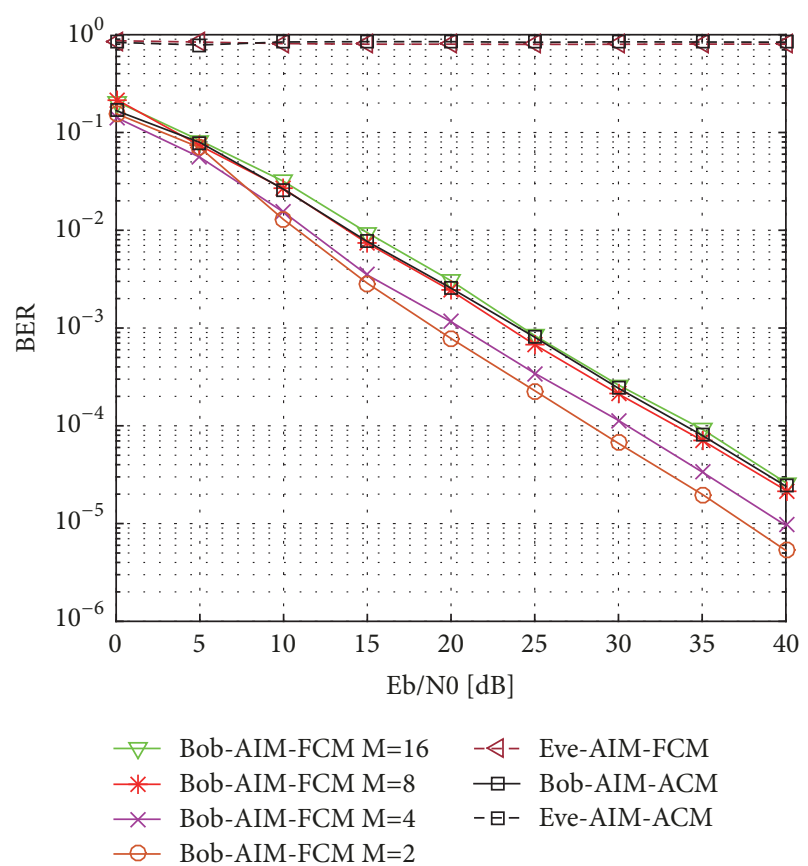

FIGURE 10: BER performance for OFDM-AIM-FCM and OFDMAIM-ACM.

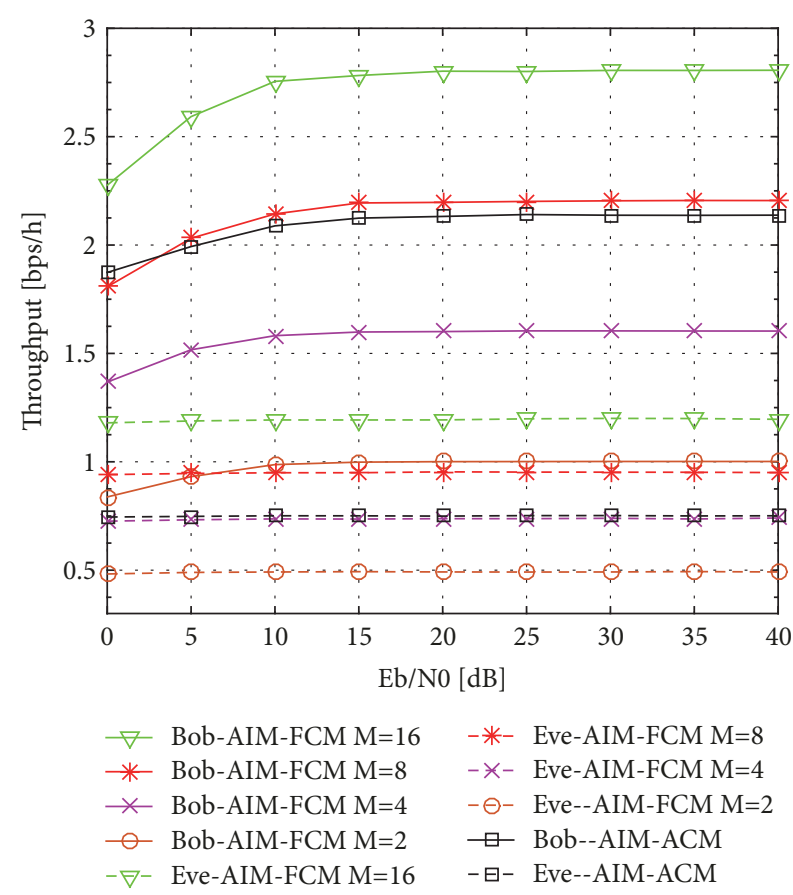

FIGURE 11: Throughput performance for OFDM-AIM-FCM and OFDM-AIM-ACM.

$M=8$, and $M=16$. Similarly, Figure 11 also presents the throughput results of our proposed OFDM-AIM-ACM based PLS technique for Bob and Eve. It is clear from Figure 11 that the throughput of OFDM-AIM-ACM is approximately similar to the case of OFDM-AIM-FCM with $M=8$ while throughput of Eve is the worst for all the values of SNR. 


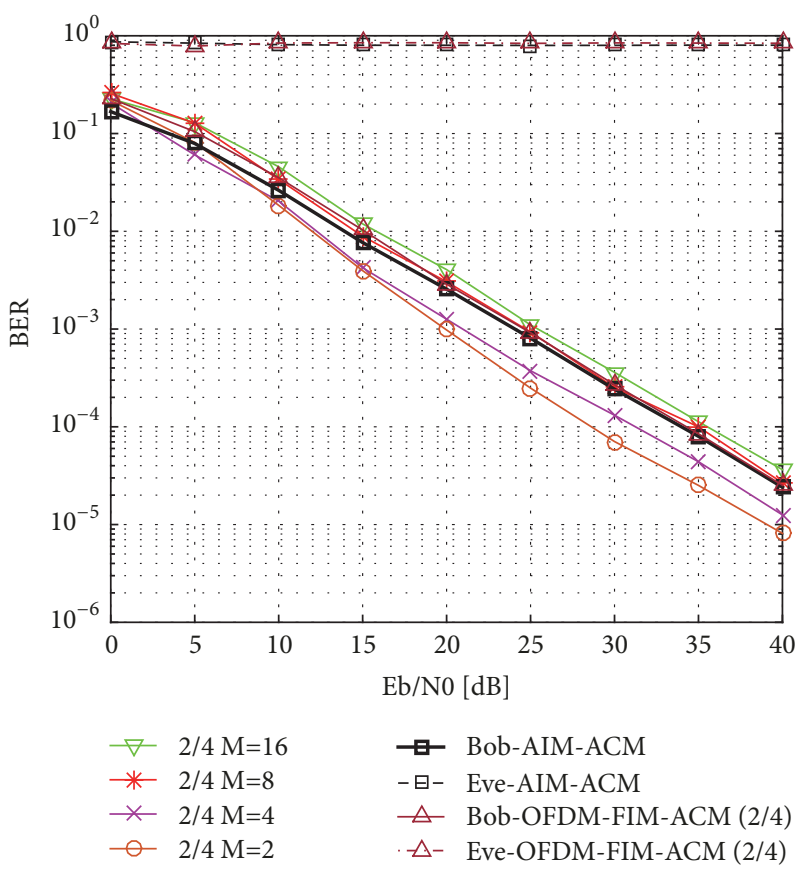

FIGURE 12: BER comparison of OFDM-IM $(n=4, k=2)$, OFDMAIM-ACM, and OFDM-FIM-ACM $(n=4, k=2)$.

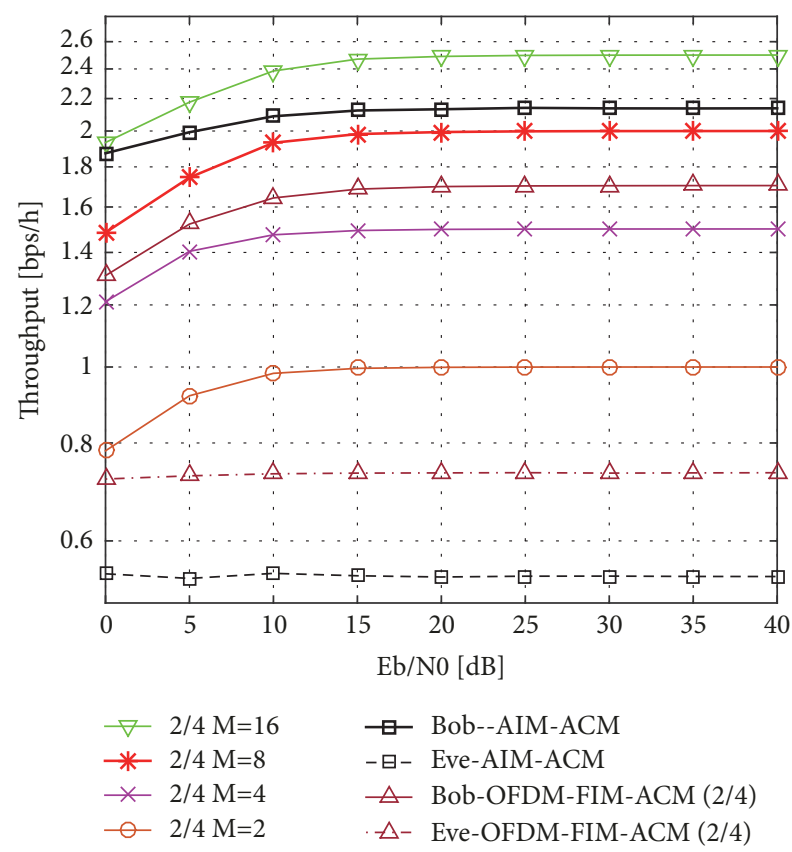

FIgURE 13: Throughput comparison of OFDM-IM $(n=4, k=2)$, OFDM-AIM-ACM, and OFDM-FIM-ACM $(n=4, k=2)$.

Figure 12 presents a comparison of BER performances between the proposed OFDM-AIM-ACM scheme and OFDM-IM ( $n=4, k=2$ ) (Ref.) with CM order of $\{2,4,8,16\}$. It is observed from Figure 12 that the BER performance of OFDM-AIM-ACM is similar to the case of OFDM-IM ( $n=4, k=2)$ (Ref.) with $M=8$. At equivalent BER, it is also noticed that the throughput of the proposed OFDM-AIM-ACM outperforms the OFDM-IM ( $n=4, k=2$ ) (Ref.) with $M=8$ at all values of SNR as presented in Figure 13. It is also observed from Figures 12 and 13 that the BER and throughput performances of Eve are the worst at all values of SNR for the proposed OFDM-AIM-ACM scheme while her BER and throughput performances are similar to that of Bob for the cases of OFDM-IM ( $n=4, k=2)$ (Ref.). Hence, the proposed scheme can enhance security and spectral efficiency jointly.

Moreover, Figures 12 and 13, respectively, also compare the BER and throughput performances of the OFDM-AIMACM scheme with the OFDM-FIM-ACM $(n=4, k=$ 2) based on [34]. It is observed from the figures that at approximately equivalent BER the proposed OFDM-AIMACM outperforms OFDM-FIM-ACM $(n=4, k=2)$ in terms of throughput. It is also observed from Figures 12 and 13 that OFDM-FIM-ACM $(n=4, k=2)$ can also provide security. Note that OFDM-AIM-ACM is more secure as compared to OFDM-AIM-FCM because in the case of OFDM-AIM-ACM both SAR and CM are varied adaptively while in case of OFDM-AIM-FCM only SAR is varied.

The below subsections present the effect of imperfect channel estimation and effect of channel correlation between Bob's channel and Eve's channel on the performances of OFDM-AIM-FCM and OFDM-AIM-ACM.

5.2.1. Security Algorithms under Imperfect Channel Estimation. In order to evaluate the robustness of the proposed security algorithms against imperfect channel estimation, intentional error is added at both the transmitter and receiver $\left(\Delta \mathbf{h}_{T / R}\right)$ to the true channel $\left(\mathbf{h}_{b}\right)$ to obtain new erroneous channels given by $\widetilde{\mathbf{h}}_{b}=\mathbf{h}_{b}+\Delta \mathbf{h}[20,35]$. The intentional error $(\Delta \mathbf{h})$ is modeled as an independent complex Gaussian noise with zero mean and variance $\left(\sigma^{2}=m s e \times 10^{-S N R_{d B} / 10}\right)$, where $m s e$ is a variable related to mean square of estimator's quality. Figures 14 and 15, respectively, present the BER performances for OFDM-AIM-FCM and OFDM-AIM-ACM under different estimation qualities with $m s e=0$ (perfect estimation), $m s e=0.02$, $m s e=0.05$, and $m s e=$ 0.1 . It is shown that imperfect channel estimation leads to small degradation in the BER performance. However, this degradation can be overcome by increasing the length or power of the training sequence. Moreover, there are some interesting algorithms proposed in the literature that can minimize the channel estimation error, such as in [1]

5.2.2. Effect of Eve's Channel Correlation with Bob's Channel. This subsection presents the effect of the correlation between the channel of legitimate receiver and Eve and evaluate the performance in terms of BER as a security metric. Firstly, the assumption of channel decorrelation requires that Bob and Eve be located at more than one-half wavelength away from Alice. This is a practical assumption in many realistic scenarios and is assumed in many prominent works in the literature (such as $[21,23,24]$ ). We have performed additional new simulations to show the effect of the correlation between the channels of legitimate receiver and eavesdropper on the 


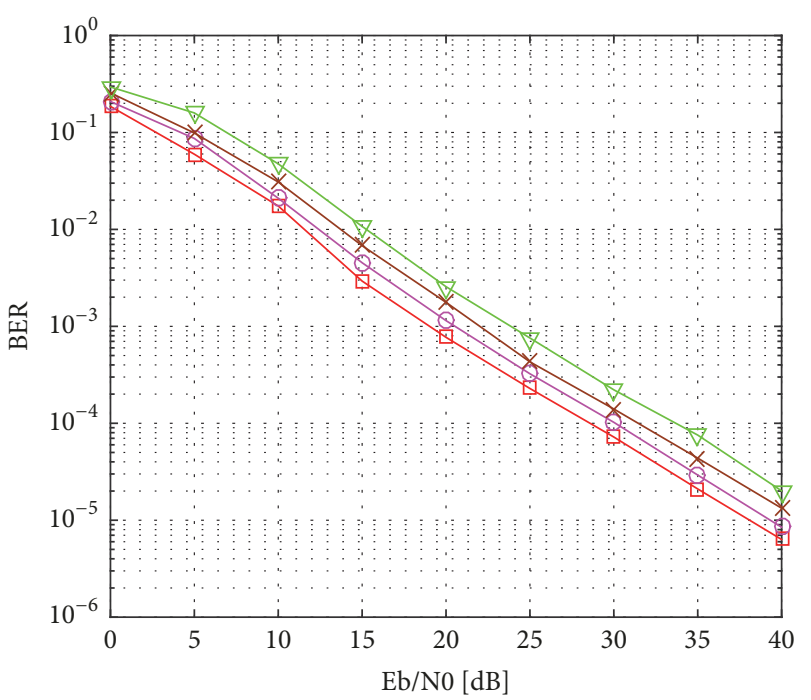

$$
\begin{array}{ll}
\square & \begin{array}{l}
\text { Bob-AIM-FCM } \\
(\mathrm{mse}=0)
\end{array} \\
\bigcirc & \mathrm{mse}=0.02 \\
\times & \mathrm{mse}=0.05 \\
\nabla & \mathrm{mse}=0.1
\end{array}
$$

FIGURE 14: BER comparison of OFDM-AIM-FCM ( $m s e=$ $0,0.02,0.05,0.1)$.

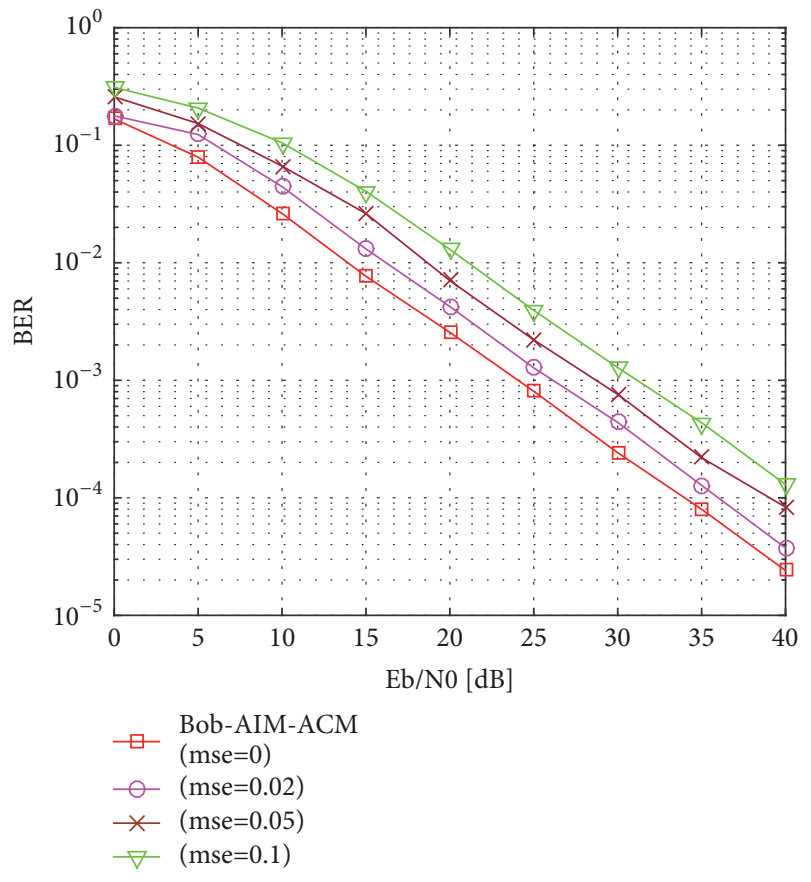

FIGURE 15: BER comparison of OFDM-AIM-ACM ( $m s e=$ $0,0.02,0.05,0.1)$.

secrecy performance that is measured in terms of BER as a security metric as explained above.

Figures 16 and 17, respectively, present the BER performances for OFDM-AIM-FCM and OFDM-AIM-ACM when Eve's channel is correlated to Bob's one. The model for channel correlation between the channels of legitimate receiver and

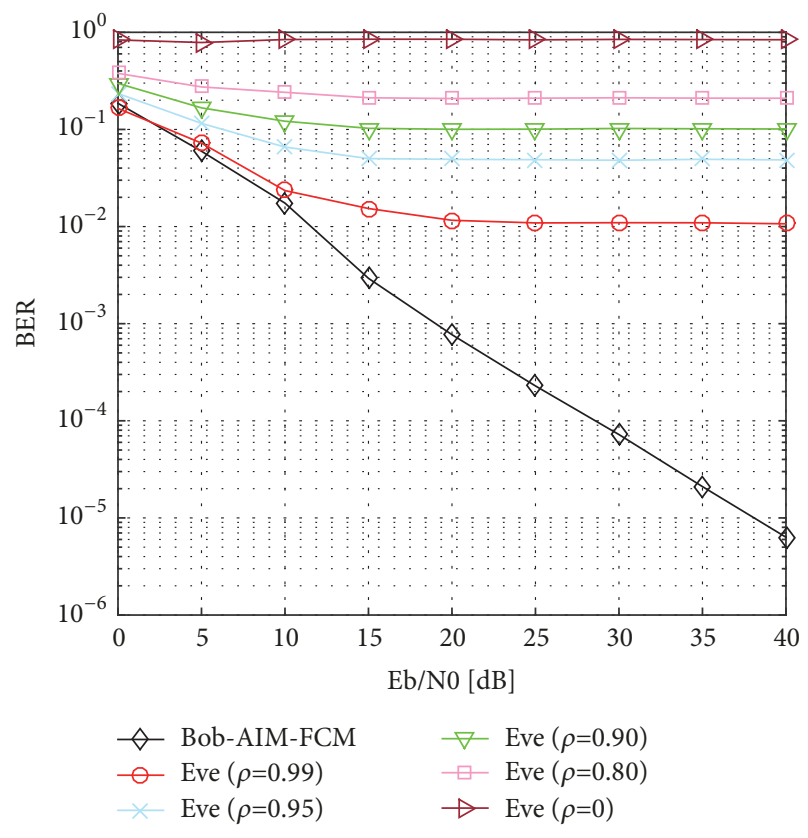

FIGURE 16: BER comparison of Bob (OFDM-AIM-FCM) and Eve with correlation coefficient $(\rho=0,0.80,0.90,0.95,0.99)$.

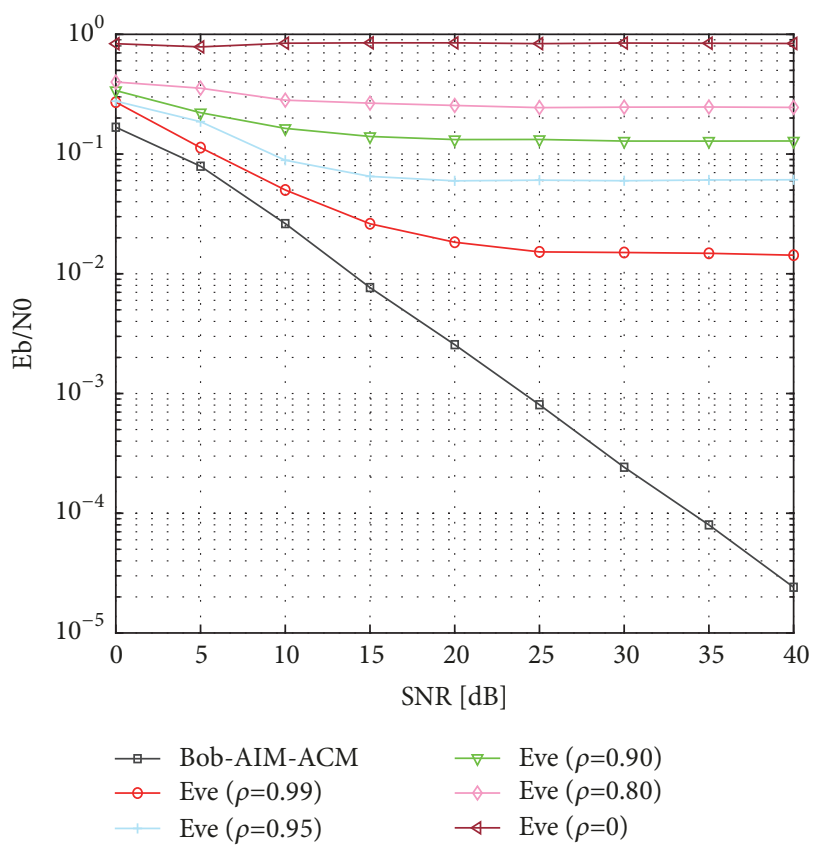

Figure 17: BER comparison of Bob (OFDM-AIM-ACM) and Eve with correlation coefficient $(\rho=0,0.80,0.90,0.95,0.99)$.

eavesdropper assumed in this work is similar to the one presented in [24] and is given as follows:

$$
\mathbf{h}_{e}=\rho \mathbf{h}_{b}+(1-\rho) \mathbf{E}
$$

where $E$ represents an independent channel while $\rho$ is the correlation factor. We present BER performance for the correlation values of $(\rho=0,0.80,0.90,0.95,0.99)$. It should be noted that even with correlation between Bob's and Eve's 


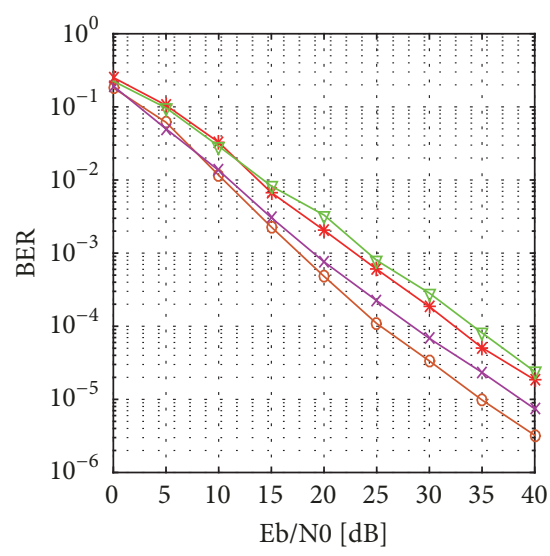

- $1 / 4 \mathrm{M}=2 \quad$ * $1 / 4 \mathrm{M}=8$

$\leftarrow 1 / 4 \mathrm{M}=4 \quad \nabla \quad 1 / 4 \mathrm{M}=16$

(a) BER versus $\mathrm{SNR}(\mathrm{SAR}=1 / 4)$
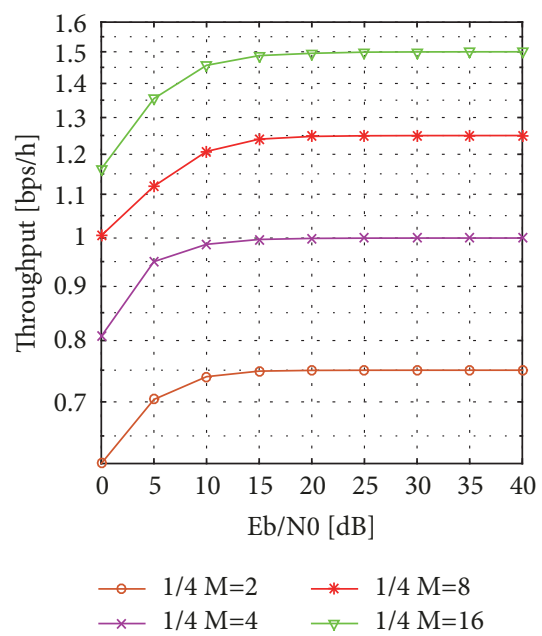

(d) Throughput versus SNR (SAR=1/4)

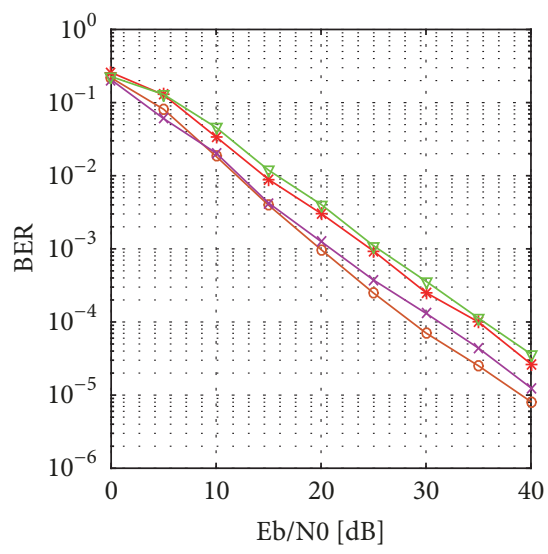

$\rightarrow 2 / 4 \mathrm{M}=2 \quad *-2 / 4 \mathrm{M}=8$

$\rightarrow 2 / 4 \mathrm{M}=4 \rightarrow 2 / 4 \mathrm{M}=16$

(b) BER versus $\mathrm{SNR}(\mathrm{SAR}=2 / 4)$
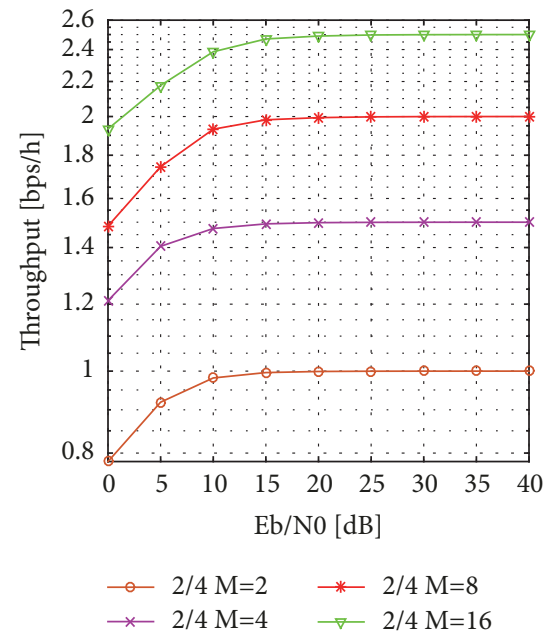

(e) Throughput versus SNR (SAR=2/4).

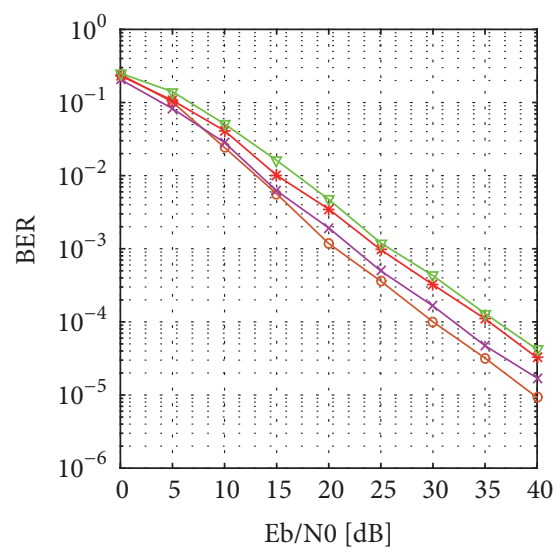

$\rightarrow 3 / 4 \mathrm{M}=2 \quad$ * $3 / 4 \mathrm{M}=8$

$\rightarrow 3 / 4 \mathrm{M}=4 \quad \nabla-3 / 4 \mathrm{M}=16$

(c) BER versus $\mathrm{SNR}(\mathrm{SAR}=3 / 4)$

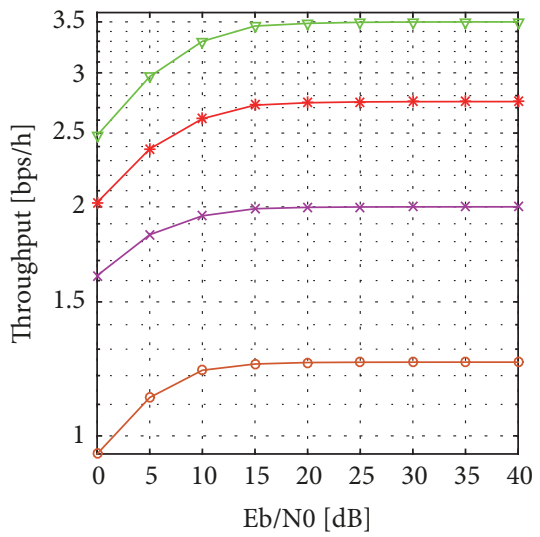

$\rightarrow 3 / 4 \mathrm{M}=2 \quad \rightarrow-3 / 4 \mathrm{M}=8$

$\rightarrow 3 / 4 \mathrm{M}=4 \quad \rightarrow 3 / 4 \mathrm{M}=16$

(f) Throughput versus SNR (SAR=3/4).

FIGURE 18: OFDM-IM with SAR values of $(1 / 4,2 / 4,3 / 4)$ and CM orders of $(2,4,8$, and 16).

channels, the proposed algorithms can still provide some level of QoS based security.

5.3. OFDM-VIM-VCM. Figures 18 and 19 present the extensive simulations related to OFDM-VIM-VCM scheme for QoS based communication in order to maximize the spectral efficiency. Note that the system model for this technique is the same as explained in Section 2, except the Eve link, which is not considered in this case. The basic concept is to vary the SAR and CM with the increase in SNR to maximize the spectral efficiency while fulfilling certain QoS requirement. In this approach, BER and throughput curves for four types of CM order, such as $M=2, M=4, M=8$, and $M=16$, are implemented for each of SAR types, such as $1 / 4,2 / 4,3 / 4$, and 4/4, and presented in Figures 18, 19(a), and 19(d). Afterwards, certain curves are selected based on OFDM-VIM-VCM for QoS based communication.

In Figure 19(b), we merge the BER curves of SAR values of $1 / 4,2 / 4,3 / 4$, and $4 / 4$ for CM order of $M=2, M=4$, $M=8$, and $M=16$. Similarly, in Figure 19(e), throughput curves of SAR values of $1 / 4,2 / 4,3 / 4$, and $4 / 4$ for $\mathrm{CM}$ order of $M=2, M=4, M=8$, and $M=16$ are also merged.

Afterwards, among the BER curves of Figure 19(b) that have similar performance, we select a curve that has maximum value of throughput. From the selected curves in the former step, we select those curves that have a performance gap among them. Finally, the resultant curves are presented in Figure 19(c). Afterwards, the corresponding throughput curves of Figure 19(e) related to selected BER curves in Figure 19(c) are also selected and presented in Figure 19(f).

Based on Figures 19(c) and 19(f), we develop a switching tables for QoS based communication in order to maximize the throughput. In this work, as an example, switching among different modulation types based on the SNR for the case of BER $<10^{-3}$ and BER $<10^{-4}$ is presented in Table 6 . The table depicts different SAR and CM values of system for different SNR ranges to maximize the spectral efficiency while fulfilling different QoS requirements. Afterwards, this table can be used for different QoS based communication services for maximizing spectral efficiency in a similar way 

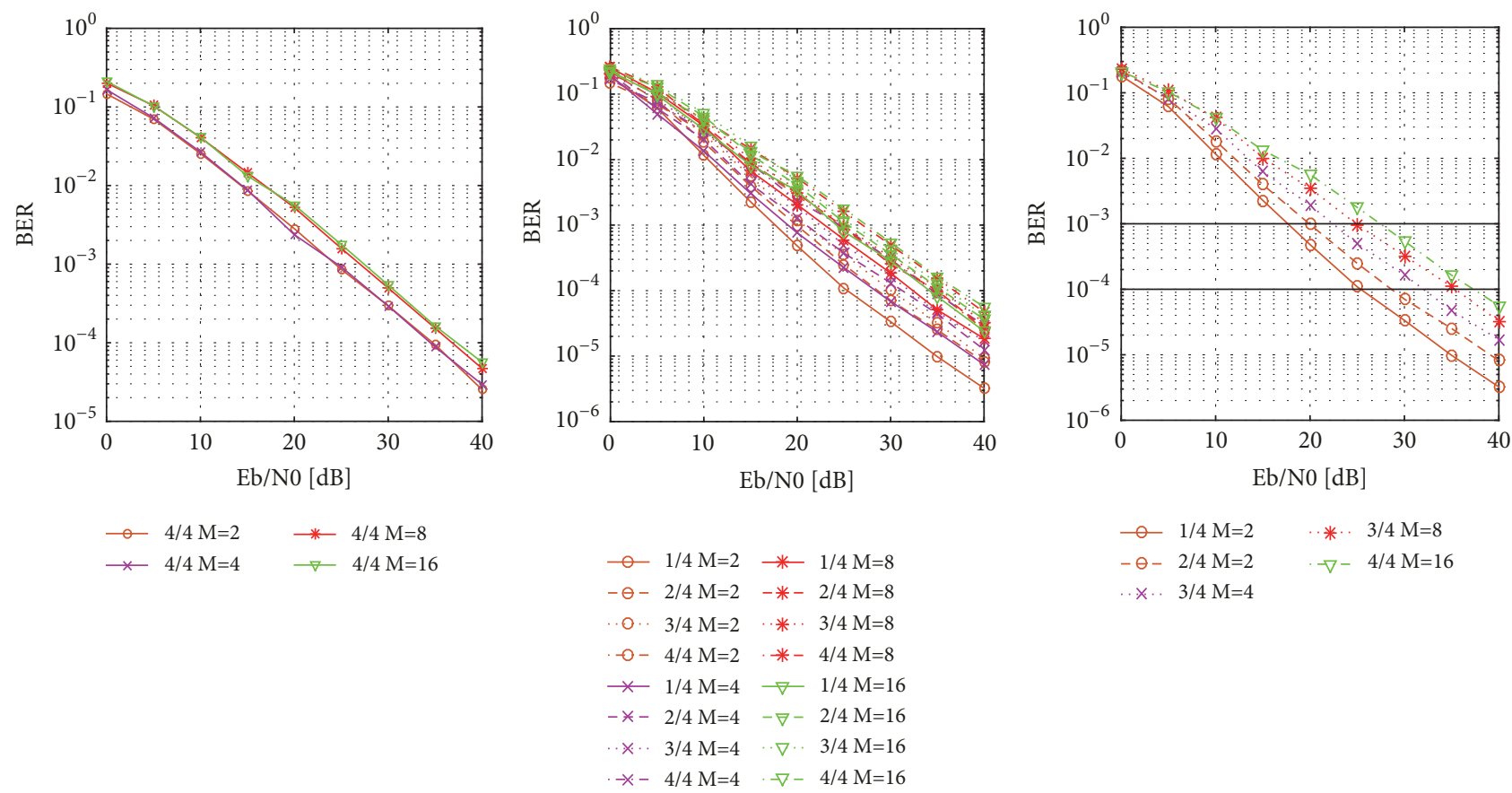

(a) BER versus $\mathrm{SNR}(\mathrm{SAR}=4 / 4)$

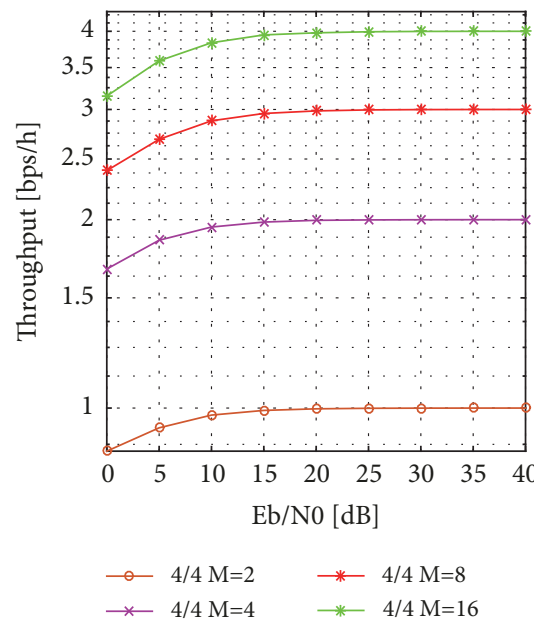

(d) Throughput versus SNR (SAR=4/4)

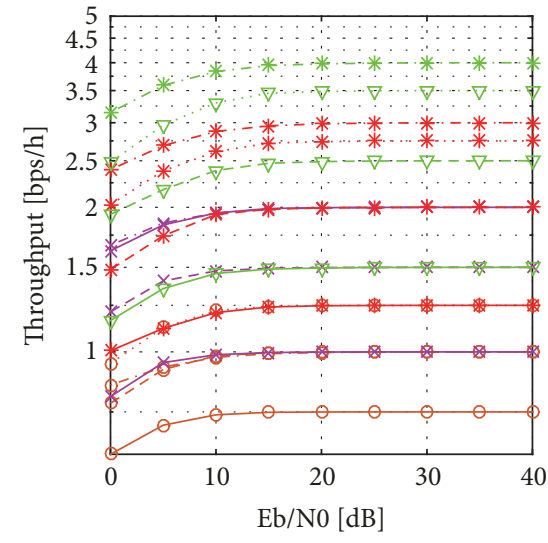

$$
\begin{aligned}
& \text { - } 1 / 4 \mathrm{M}=2 \rightarrow 1 / 4 \mathrm{M}=8 \\
& -\ominus-2 / 4 \mathrm{M}=2 \quad-*-2 / 4 \mathrm{M}=8 \\
& \text {. o. } 3 / 4 \mathrm{M}=2 \text {.**. } 3 / 4 \mathrm{M}=8 \\
& -0-4 / 4 \mathrm{M}=2 \quad *-4 / 4 \mathrm{M}=8 \\
& \times 1 / 4 \mathrm{M}=4-\nabla-1 / 4 \mathrm{M}=16 \\
& -*-2 / 4 \mathrm{M}=4 \quad-\nabla-2 / 4 \mathrm{M}=16
\end{aligned}
$$

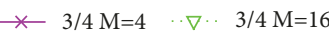

$$
\begin{aligned}
& -*-4 / 4 \mathrm{M}=4 \quad *-4 / 4 \mathrm{M}=16
\end{aligned}
$$

(e) Throughput versus SNR (merged)

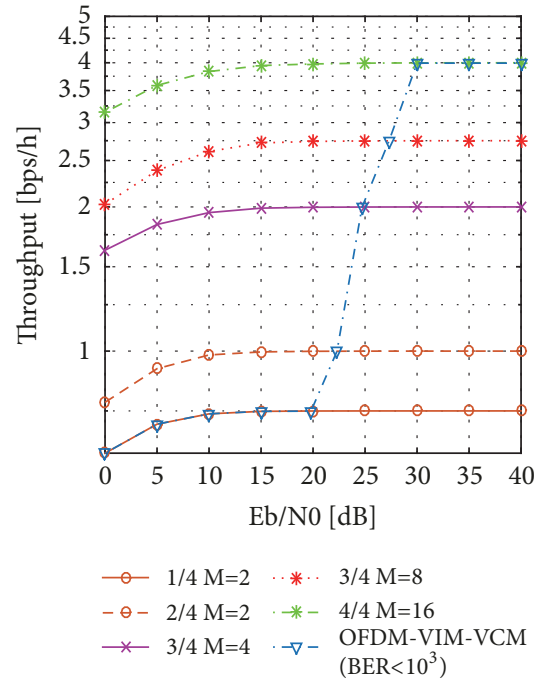

(f) Throughput versus SNR (selective)

FIGURE 19: OFDM-IM with SAR value of (4/4) and CM orders of $(2, M=4, M=8$, and $M=16)$, merged curves for different cases of OFDM-IM and selected curves for different cases of OFDM-IM for QoS based communication.

as performed in [16]. The result of OFDM-VIM-VCM for the case of $B E R<10^{-3}$ is presented in Figure 19(f).

\section{Conclusion}

In this work, effective algorithms that change SAR and/or CM adaptively in each subblock of the OFDM-IM scheme based on the channel characteristics of the legitimate receiver are proposed for enhancing PLS and SE. Particularly, the first two algorithms named as OFDM-AIM-FCM and OFDM-AIMACM are designed for enhancing PLS and SE, while the third algorithm named as OFDM-VIM-VCM is designed for QoS based communication for enhancing SE. Simulation results show that the first two algorithms can provide significant security enhancement whereas the third algorithm ensures 
TABLE 6: Switching table for OFDM-VIM-VCM.

(a) $B E R<10^{-3}$

\begin{tabular}{lcc}
\hline $\mathrm{Eb} / \mathrm{NO}(\mathrm{E})$ & $\mathrm{M}$ & $\mathrm{BER}<10^{-3}$ \\
\hline $17.6<\mathrm{E}<19.9$ & 2 & $\mathrm{SAR}$ \\
$19.9<\mathrm{E}<22.4$ & 2 & $1 / 4$ \\
$22.4<\mathrm{E}<24.8$ & 4 & $2 / 4$ \\
$24.8<\mathrm{E}<27.4$ & 8 & $3 / 4$ \\
$27.4<\mathrm{E}$ & 16 & $3 / 4$ \\
\hline
\end{tabular}

(b) $B E R<10^{-4}$

\begin{tabular}{lcc}
\hline & \multicolumn{3}{c}{$\mathrm{BER}<10^{-4}$} \\
$\mathrm{~Eb} / \mathrm{NO}(\mathrm{E})$ & $\mathrm{M}$ & $\mathrm{SAR}$ \\
\hline $25.3<\mathrm{E}<28.6$ & 2 & $1 / 4$ \\
$28.6<\mathrm{E}<32$ & 2 & $2 / 4$ \\
$32<\mathrm{E}<35.3$ & 4 & $3 / 4$ \\
$35.3<\mathrm{E}<37.3$ & 8 & $3 / 4$ \\
$37.3<\mathrm{E}$ & 16 & $4 / 4$ \\
\hline
\end{tabular}

QoS based communication aiming to maximize spectral efficiency.

\section{Data Availability}

The data used to support the findings of this study are included within the article.

\section{Conflicts of Interest}

The authors declare that there are no conflicts of interest regarding the publication of this paper.

\section{References}

[1] J. M. Hamamreh, H. M. Furqan, and H. Arslan, "Secure pre-coding and post-coding for OFDM systems along with hardware implementation," in Proceedings of the 2017 13th International Wireless Communications and Mobile Computing Conference (IWCMC), pp. 1338-1343, Valencia, Spain, June 2017.

[2] H. M. Furqan, J. M. Hamamreh, and H. Arslan, "Secret key generation using channel quantization with SVD for reciprocal MIMO channels," in Proceedings of the 13th International Symposium on Wireless Communication Systems, ISWCS 2016, pp. 597-602, Poland, September 2016.

[3] Y. Liu, H. H. Chen, and L. Wang, "Physical layer security for next generation wireless networks: Theories, technologies, and challenges," in IEEE Communications Surveys Tutorials, vol. 19, no. 1, pp. 347-376, 2017.

[4] X. Chen, D. W. K. Ng, W. H. Gerstacker, and H. H. Chen, "A Survey on multiple-antenna techniques for physical layer security," ГГ in IEEE Communications Surveys Tutorials, vol. 19, no. 2, pp. 1027-1053, 2017.

[5] E. Basar, M. Wen, R. Mesleh, M. Di Renzo, Y. Xiao, and H. Haas, "Index Modulation Techniques for Next-Generation Wireless Networks," IEEE Access, vol. 5, pp. 16693-16746, 2017.
[6] E. g. Basar, U. Aygolu, E. Panayirci, and H. V. Poor, "Orthogonal frequency division multiplexing with index modulation," IEEE Transactions on Signal Processing, vol. 61, no. 22, pp. 5536-5549, 2013.

[7] J. Choi, "Coded OFDM-IM with Transmit Diversity", IEEE Transactions on Communications, vol. 65, no. 7, pp. 3164-3171, 2017.

[8] J. Li, M. Wen, X. Cheng, Y. Yan, S. Song, and M. H. Lee, "Generalized Precoding-Aided Quadrature Spatial Modulation," IEEE Transactions on Vehicular Technology, vol. 66, no. 2, pp. 18811886, 2017.

[9] M. Wen, E. Basar, Q. Li, B. Zheng, and M. Zhang, "MultipleMode Orthogonal Frequency Division Multiplexing with Index Modulation," IEEE Transactions on Communications, vol. 65, no. 9, pp. 3892-3906, 2017.

[10] E. Soujeri, G. Kaddoum, M. Au, and M. Herceg, "Frequency Index Modulation for Low Complexity Low Energy Communication Networks," IEEE Access, vol. 5, pp. 23276-23287, 2017.

[11] M. Au, G. Kaddoum, S. Francois, and S. Ebrahim, "A Joint Code-Frequency Index Modulation for Low-complexity, High Spectral and Energy Efficiency Communications," https:// arxiv.org/abs/1712.07951.

[12] E. Soujeri, G. Kaddoum, and M. Herceg, "Design of an initial condition-index chaos shift keying modulation," IEEE Electronics Letters, vol. 54, no. 7, pp. 447-449, 2018.

[13] Q. Wang, H. Su, K. Ren, and K. Kim, "Fast and scalable secret key generation exploiting channel phase randomness in wireless networks," in Proceedings of the IEEE INFOCOM, pp. 1422-1430, Shanghai, China, April 2011.

[14] H. Qin, Y. Sun, T.-H. Chang et al., "Power allocation and timedomain artificial noise design for wiretap OFDM with discrete inputs," IEEE Transactions on Wireless Communications, vol. 12, no. 6, pp. 2717-2729, 2013.

[15] Z. E. Ankaral, M. Karabacak, and H. Arslan, "Cyclic Feature Concealing CP Selection for Physical Layer Security," in Proceedings of the 2014 IEEE Military Communications Conference (MILCOM), pp. 485-489, Baltimore, MD, USA, October 2014.

[16] J. M. Hamamreh, M. Yusuf, T. Baykas, and H. Arslan, "Cross MAC/PHY layer security design using ARQ with MRC and adaptive modulation," in Proceedings of the 2016 IEEE Wireless Communications and Networking Conference, WCNC 2016, Qatar, April 2016.

[17] E. Guvenkaya and H. Arslan, "Secure communication in frequency selective channels with fade-avoiding subchannel usage," in Proceedings of the 2014 IEEE International Conference on Communications Workshops, ICC 2014, pp. 813-818, Australia, June 2014.

[18] D. W. Ng, E. S. Lo, and R. Schober, "Energy-Efficient Resource Allocation for Secure OFDMA Systems," IEEE Transactions on Vehicular Technology, vol. 61, no. 6, pp. 2572-2585, 2012.

[19] H. M. Furqan, J. M. Hamamreh, and H. Arslan, "Enhancing physical layer security of OFDM systems using channel shortening," in Proceedings of the 2017 IEEE 28th Annual International Symposium on Personal, Indoor, and Mobile Radio Communications (PIMRC), pp. 1-5, Montreal, QC, October 2017.

[20] J. M. Hamamreh, E. Basar, and H. Arslan, "OFDM-Subcarrier Index Selection for Enhancing Security and Reliability of 5G URLLC Services," IEEE Access, 2017. 
[21] F. Wu, R. Zhang, L.-L. Yang, and W. Wang, “Transmitter precoding-aided spatial modulation for secrecy communications," IEEE Transactions on Vehicular Technology, vol. 65, no. 1, pp. 467-471, 2016.

[22] L. Wang, S. Bashar, Y. Wei, and R. Li, "Secrecy Enhancement Analysis Against Unknown Eavesdropping in Spatial Modulation," IEEE Communications Letters, vol. 19, no. 8, pp. 1351-1354, 2015.

[23] X. Wang, X. Wang, and L. Sun, "Spatial modulation aided physical layer security enhancement for fading wiretap channels," in Proceedings of the 2016 8th International Conference on Wireless Communications \& Signal Processing (WCSP), pp. 1-5, Yangzhou, China, October 2016.

[24] X. Jiang, M. Wen, H. Hai, J. Li, and S. Kim, "Secrecy-Enhancing Scheme for Spatial Modulation," IEEE Communications Letters, vol. 22, no. 3, pp. 550-553, 2018.

[25] Y. Lee, H. Jo, Y. Ko, and J. Choi, "Secure Index and Data Symbol Modulation for OFDM-IM," IEEE Access, vol. 5, pp. 2495924974, 2017.

[26] "3GPP, Policy and charging control architecture, 3GPP Std. TS 23.203 V11.6.0," 2012, http://www.qtc.jp/3GPP/Specs/23203b60.pdf.

[27] P. Robertson, E. Villebrun, and P. Hoeher, "A comparison of optimal and sub-optimal MAP decoding algorithms operating in the log domain," in Proceedings of the IEEE International Conference on Communications (ICC '95), vol. 2, pp. 1009-1013, IEEE, Seattle, Wash, USA, June 1995.

[28] H. Jafarkhani, Space-Time Coding, Cambridge University Press, Cambridge, 2005.

[29] R. A. Horn and C. R. Johnson, Matrix Analysis, Cambridge University Press, Cambridge, UK, 1985.

[30] H. Li, X. Wang, and J.-Y. Chouinard, "Eavesdropping-resilient OFDM system using sorted subcarrier interleaving," IEEE Transactions on Wireless Communications, vol. 14, no. 2, pp. 1155-1165, 2015.

[31] E. Güvenkaya, J. M. Hamamreh, and H. Arslan, "On physicallayer concepts and metrics in secure signal transmission," Physical Communication, vol. 25, pp. 14-25, 2017.

[32] J. M. Hamamreh and H. Arslan, "Joint PHY/MAC Layer Security Design Using ARQ with MRC and Null-Space Independent, PAPR-Aware Artificial Noise in SISO Systems," IEEE Transactions on Wireless Communications, pp. 1-1.

[33] ICAO, "Uniting Aviation," in Proceedings of the AN-Conf/13 - ICAO Thirteenth - Air Navigation Conference, Montereal, Canada, Oct. 2018, https://www.icao.int.

[34] J. Faezah and K. Sabira, "Adaptive modulation for OFDM systems," International Journal of Communication Networks and Information Security, vol. 1, no. 2, pp. 1-8, 2009.

[35] J. M. Hamamreh and H. Arslan, "Secure Orthogonal Transform Division Multiplexing (OTDM) Waveform for 5G and beyond," IEEE Communications Letters, vol. 21, no. 5, pp. 1191-1194, 2017. 


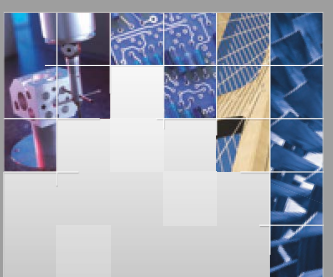

\section{Enfincering}
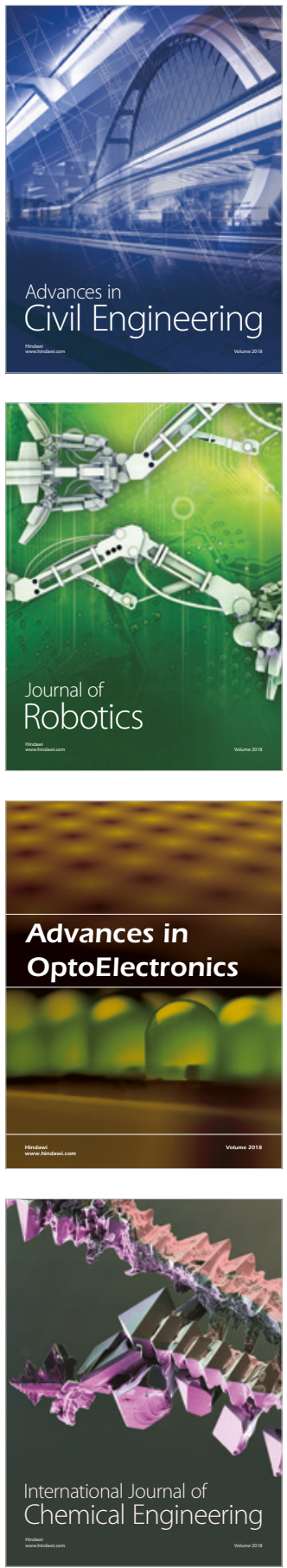

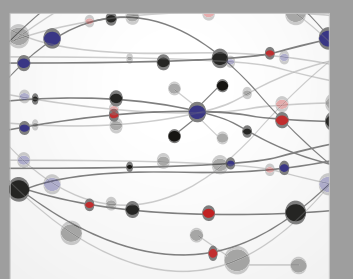

\section{Rotating \\ Machinery}

The Scientific World Journal

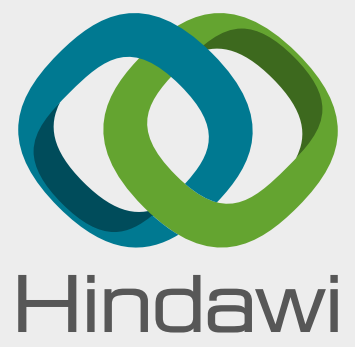

Submit your manuscripts at

www.hindawi.com
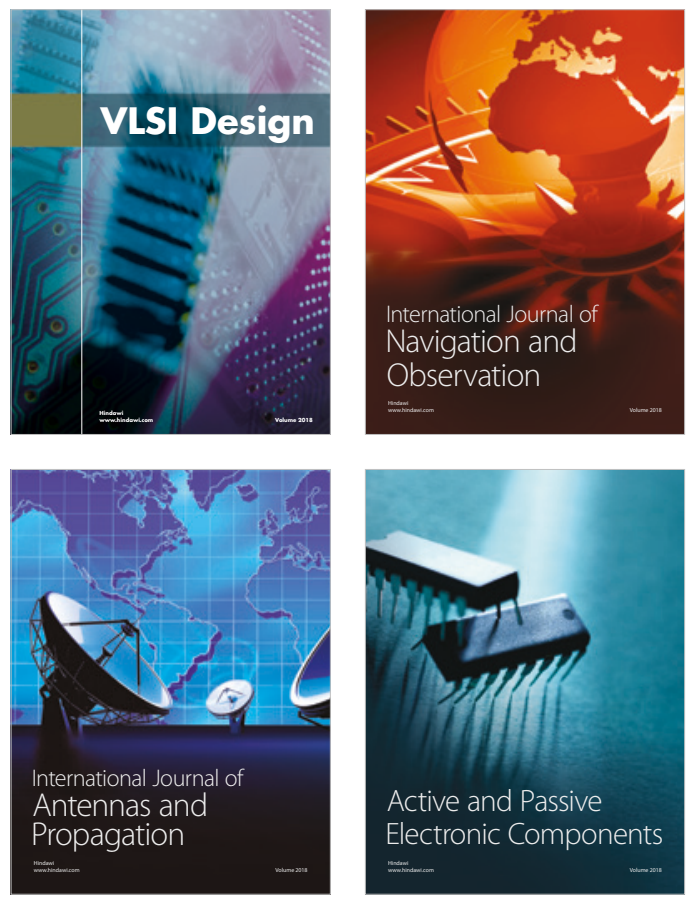
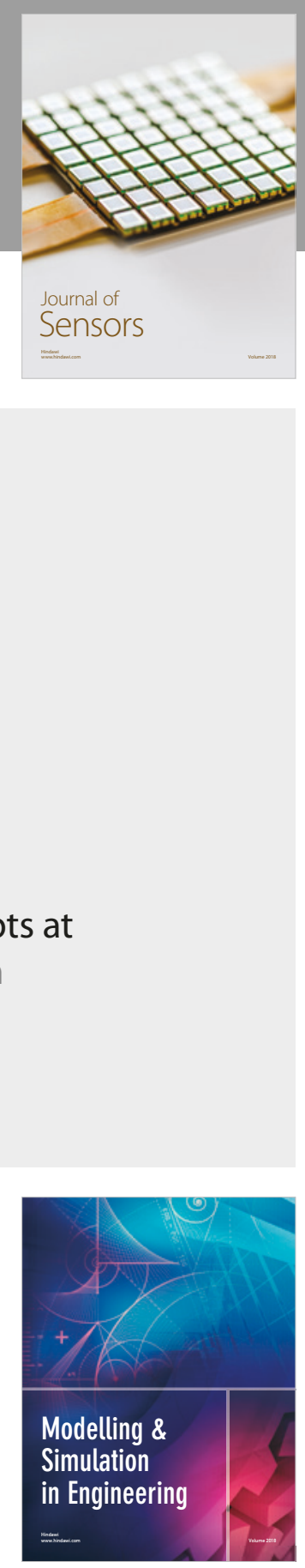

\section{Advances \\ Multimedia}
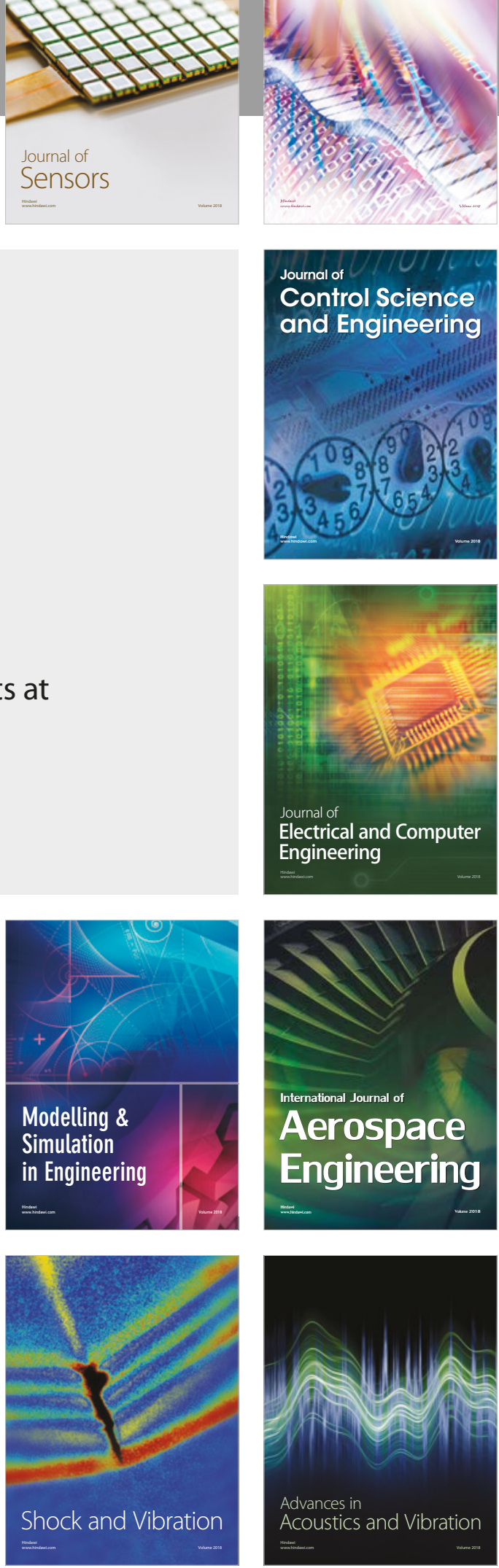